\title{
Throughput Scaling in Cognitive Multiple Access With Average Power and Interference Constraints
}

\author{
Ehsan Nekouei, Student Member, IEEE, Hazer Inaltekin, Member, IEEE, and Subhrakanti Dey, Senior Member, IEEE
}

\begin{abstract}
This paper derives tight ergodic sum-rate capacity scaling limits for cognitive radio secondary networks under five different communication environments (CoE) for two different network types when secondary users' (SUs) transmission powers are optimally allocated. The network types studied are power-interference limited (PIL) networks and interference limited (IL) networks. In PIL networks, SUs' transmissions are limited by both an average total power constraint and a constraint on the average interference that they cause to primary users (PUs). In IL networks, SUs' transmissions are only limited by an average interference constraint. The capacity scaling results in PIL networks are derived for three different $\mathrm{CoEs}$ in which secondary transmitter to secondary base station (STSB) channel gains are Rayleigh distributed while secondary transmitter to primary base station (STPB) channel gains are Rayleigh, Rician or Nakagami distributed. It is shown that secondary network capacity scales according to $\log \log (N)$ in these three CoEs, where $N$ is the number of SUs. In addition to these three CoEs, two more CoEs are also studied for IL networks: Rician or Nakagami distributed STSB channel gains and Rayleigh distributed STPB channel gains. It is shown that the secondary network capacity scales according to $\log (N)$ for all five CoEs in IL networks. This result implies exponential capacity gains in IL networks over PIL networks. The same capacity scaling results are shown to hold even for heterogeneous cognitive radio networks in which different SUs experience statistically different channel conditions. In some cases, our analysis leads to a new notion called effective number of users, which signifies the effective number of users contributing to multiuser diversity in cognitive radio networks. For example, effective number of users is given by $\frac{K+1}{\mathrm{e}} N$ when STSB channel gains are Rayleigh distributed and STPB channel gains are Rician distributed with a Rician factor $K$.
\end{abstract}

Index Terms-Cognitive radio networks, multiple access, multiuser diversity, sum capacity.

\section{INTRODUCTION}

\section{A. Background and Motivation}

W ITH the advent of $4 \mathrm{G}$ wireless networks striving for ever higher data rates, efficient use of spectrum and its scarcity are expected to be major (and bigger) design challenges to overcome in future wireless communication systems

Manuscript received March 08, 2011; revised August 07, 2011; accepted October 10, 2011. Date of publication October 28, 2011; date of current version January 13,2012 . The associate editor coordinating the review of this manuscript and approving it for publication was Dr. Milica Stojanovic.

E. Nekouei and S. Dey are with the Department of Electrical and Electronic Engineering, The University of Melbourne, VIC 3010, Australia (e-mail: e.nekouei@pgrad.unimelb.edu.au; hazeri@unimelb.edu.au; sdey@unimelb.edu.au).

H. Inaltekin is with the Department of Electrical and Electronics Engineering, Antalya International University, Antalya, Turkey (e-mail: hazeri@ antalya.edu. tr).

Color versions of one or more of the figures in this paper are available online at http://ieeexplore.ieee.org.

Digital Object Identifier 10.1109/TSP.2011.2174054
[1]. Cognitive radio technology empowered with radio etiquette protocols (e.g., IEEE 802.22) has been proposed as a possible solution to the spectrum scarcity and the problem of efficient spectrum utilization by allowing unlicensed users (alternatively called: secondary users or SUs) to access the spectrum reserved for licensed users (alternatively called: primary users or PUs), e.g., see [2] and [3]. The rationale is that SUs can use this spectrum as long as they do not cause harmful degradation to the primary transmission. Potential spectrum utilization gains to be achieved by the enablement of such cognitive radio technologies can be best assessed only when we have theoretical limits on data rates that can be delivered to SUs subject to various constraints imposed by cognitive radio etiquette protocols mediating transmissions. To this end, this paper contributes a suite of results to theoretical limits on achievable aggregate data rates in cognitive radio networks by providing tight capacity scaling laws, as a function of the number of SUs, under transmission power and interference constraints for various wireless channel models.

Various paradigms for the interoperability of PUs and SUs in a cognitive radio setting have been proposed such as underlay, overlay and interweave [4]. The paradigm of interest in the current paper is the underlay model, also known as the spectrum sharing scenario, for cognitive radio networks. In this model, SUs share the spectrum with PUs regardless of PUs' ON/OFF status as long as the interference caused by them to primary receivers is kept low to guarantee a required level of Quality-ofService (QoS) on the primary network. Federal Communications Commission (FCC) in the United States introduced a key metric called interference temperature to measure the level of interference at PUs for such spectrum sharing communication scenarios [5]. In this paper, we will use the terms interference power and interference temperature interchangeably with the understanding that these two metrics are directly related to each other [6]. Our starting point to investigate secondary network capacity scaling limits here will be the jointly optimal power-control and spectrum sharing policy subject to average interference temperature constraints at PUs (as well as average transmission power constraints at SUs) mandated by a radio etiquette protocol to mediate SUs' transmissions.

Optimum cognitive spectrum sharing and power-control mechanisms as well as fundamental performance limits subject to various types of interference constraints and related QoS performance metrics such as average or peak interference constraints, primary capacity loss constraints and primary outage probability constraints have attracted considerable attention over the last five years, e.g., see [7]-[11]. Recently, optimal power allocation for ergodic sum capacity maximization in 
TABLE I

STSB AND STPB CHANNEL MODELS FOR DIFFERENT COES

\begin{tabular}{lcc}
\hline \multirow{2}{*}{ Communication Environment } & \multicolumn{2}{c}{ Channel Model } \\
\cline { 2 - 3 } & STSB Channel Model & STPB Channel Model \\
\hline First $\mathrm{CoE}\left(\mathrm{CoE}^{1}\right.$ or $\left.\mathrm{CoE}^{1-h}\right)$ & Rayleigh & Rician- $K$ \\
\hline Second $\mathrm{CoE}\left(\mathrm{CoE}^{2}\right.$ or $\left.\mathrm{CoE}^{2-h}\right)$ & Rayleigh & Rayleigh \\
\hline Third $\mathrm{CoE}\left(\mathrm{CoE}^{3}\right.$ or $\left.\mathrm{CoE}^{3-\mathrm{h}}\right)$ & Rayleigh & Nakagami- $m$ \\
\hline Fourth $\mathrm{CoE}\left(\mathrm{CoE}^{4}\right.$ or $\left.\mathrm{CoE}^{4-h}\right)$ & Nakagami- $m$ & Rayleigh \\
\hline Fifth $\mathrm{CoE}\left(\mathrm{CoE}^{5}\right.$ or $\left.\mathrm{CoE}^{5-h}\right)$ & Rician- $K$ & Rayleigh \\
\hline
\end{tabular}

a multiple access and broadcast secondary network under various combinations of transmission power and interference constraints have been obtained in [10]. Among many other results, Zhang et al. in [10] showed that the jointly optimal spectrum sharing and power-control policy maximizing information theoretic sum-rate capacity of a multiple access secondary network with average transmission and interference temperature constraints for continuous fading distributions is to schedule the SU with the best joint power and interference channel state, in a sense that will be made precise in Section III, according to a power allocation policy in the form of a water-filling algorithm with changing water levels. These results are reminiscent of opportunistic scheduling type of results derived for primary networks in [12] and [13] (for multiple access channels) and [14] (for broadcast channels), and automatically trigger the need for a thorough investigation of multiuser diversity or sum-rate scaling analysis in cognitive radio multiple access or broadcast networks with increasing numbers of SUs under various forms of transmission power and interference constraints. This motivates our investigation of sum-rate scaling analysis for cognitive radio multiple access networks in this paper. In addition to various engineering insights and design ideas, our analysis primarily leads to tight capacity scaling limits for secondary multiple access networks under the jointly optimal spectrum sharing and power-control policy for different wireless channel and network models.

\section{B. Contributions}

Our main contribution in this paper is the derivation of tight secondary network capacity scaling laws under five different CoEs for two different cognitive radio network types, referred to as PIL and IL networks, when transmission powers of SUs are optimally allocated. In PIL networks, transmission powers of SUs are limited by both an average total transmission power constraint (on the secondary network) and an average interference power constraint (on the primary network). Note that such average total power constraints have been employed to study the capacity of multiple access and broadcast primary networks (e.g., see [17] and [18]) using duality theory. In IL networks, SUs' transmissions are only limited by an average interference power constraint. We refer the reader to Table I for the statistical channel models used in each CoE.

We start our analysis with CoEs in which statistical models for STSB and STPB channel gains are given as in Table I with the same parameters for all SUs, i.e., all STSB channels and all
STPB channels experience statistically identical channel conditions, although we allow the STSB channels to have a different fading distribution from the STPB channels. The asymmetry in fading distribution between STSB channels and STPB channels is motivated by the fact that the secondary network and the primary network may experience different fading conditions, due to various factors such as different physical locations, network infrastructure, user mobility pattern and so on. For example, the STSB channels may experience a rich scattering environment (i.e., Rayleigh fading) whereas the STPB channels may have a direct line of sight (i.e., Rician fading). These different $\mathrm{CoEs}$ are indexed by $\mathrm{CoE}^{i}$ for $i \in\{1,2, \ldots, 5\}$. For PIL networks under $\mathrm{CoE}^{1}, \mathrm{CoE}^{2}$, and $\mathrm{CoE}^{3}$, we show that the secondary network sum-rate capacity scales according to $\log \log (N)$ with the number of SUs $N$ (Theorem 1). For IL networks under $\mathrm{CoE}^{i}$ for $i=1,2,4$ and 5 , we show that the secondary network sum-rate capacity scales according to $\log (N)$ (Theorem 2). The scaling law for IL networks under $\mathrm{CoE}^{3}$ is given by $\frac{1}{m} \log (N)$, where $m$ is the Nakagami- $m$ fading distribution parameter (Theorem 2 ). Secondary network capacity scaling laws for PIL networks under $\mathrm{CoE}^{4}$ and $\mathrm{CoE}^{5}$ are still unknown. Our results are summarized in Table II.

From a practical point of view, these results provide benchmark curves for performance comparison and crucial insights into achievable rates with the enablement of cognitive radio technologies in next generation wireless systems. Moreover, they also indicate that an exponential gain in secondary network data rates is possible by relaxing the power limitation on the secondary network. From a theoretical point of view, it is a common practice to study sum-rate capacity of multiuser communication systems either at high SNR regimes or under a large user population assumption, e.g., see [19] and [30] and references therein, to obtain a first-order approximation for the ergodic sum-rate capacity of such systems. Our results provide similar first-order approximations for the ergodic sum-rate capacity of a cognitive radio secondary network operating under average transmission power constraints and constraints on the average interference it causes to a primary network. They also show that the secondary network is capable of achieving the same throughput scaling as the primary network even with extra interference temperature constraints.

In the process of obtaining first-order approximations for the secondary network sum-rate capacity, second-order effects, such as the effects of the fading distribution parameters, on the multiuser diversity gains are often lost, and not visible in the limit for an infinite SU population. However, these effects can be important for understanding achievable data rates in finite cognitive radio networks. To this end, our analysis in some cases leads to a new concept called effective number of users, which signifies the effective number of SUs contributing to multiuser diversity gains for finite systems, through a careful investigation of the effects of fading distribution parameters on the sum-rate capacity scaling. In particular, effective number of users is given by $\frac{K+1}{\mathrm{e}^{K}} N$ for PIL and IL networks under $\mathrm{CoE}^{1}$, while it is given by $m N^{\frac{1}{m}}$ for IL networks under $\mathrm{CoE}^{3}$, where $K$ and $m$ are parameters for the Rician and the Nakagami fading distributions in $\mathrm{CoE}^{1}$ and $\mathrm{CoE}^{3}$, respectively. Furthermore, it is observed that the multiuser diversity gain (MDG) is 
TABLE II

Throughrut SCALING IN THE COGNITIVE RADIO NETWORK

\begin{tabular}{ccccc}
\hline \multicolumn{2}{c}{ Channel Model } & & \multicolumn{2}{c}{ Network Model } \\
\cline { 1 - 2 } STSB Channel Model & STPB Channel Model & & Power-Interference Limited & Interference Limited \\
\hline Rayleigh & Rician- $K$ & & $\lim _{N \rightarrow \infty} \frac{R_{N}}{\log \log (N)}{ }^{a}=1$ & $\lim _{N \rightarrow \infty} \frac{R_{N}}{\log (N)}=1$ \\
\hline Rayleigh & Rayleigh & $\lim _{N \rightarrow \infty} \frac{R_{N}}{\log \log (N)}=1$ & $\lim _{N \rightarrow \infty} \frac{R_{N}}{\log (N)}=1$ \\
\hline Rayleigh & Nakagami- $m$ & $\lim _{N \rightarrow \infty} \frac{R_{N}}{\log \log (N)}=1$ & $\lim _{N \rightarrow \infty} \frac{R_{N}}{\log (N)}=\frac{1}{m} b$ \\
\hline Nakagami- $m$ & Rayleigh & & $\bullet$ & $\lim _{N \rightarrow \infty} \frac{R_{N}}{\log (N)}=1$ \\
\hline Rician- $K$ & Rayleigh & $\bullet$ & $\lim _{N \rightarrow \infty} \frac{R_{N}}{\log (N)}=1$ \\
\hline
\end{tabular}

${ }^{a} R_{N}$ is the sum-rate of the secondary network with $N$ secondary users.

${ }^{b} m$ is the fading parameter for Nakagami- $m$ channel model.

not significantly affected by the fading parameters in $\mathrm{CoE}^{4}$ and $\mathrm{CoE}^{5}$ for IL networks. (See the discussion in Section IV after Theorems 1 and 2 for more details.)

Finally, we also establish sum-rate capacity scaling laws for more heterogeneous CoEs, indexed by $\mathrm{CoE}^{i-\mathrm{h}}$ for $i \in\{1,2 \ldots, 5\}$, in which different SUs experience statistically different channel conditions, i.e., parameters of the statistical models given in Table I for STSB and STPB channel gains are different for different SUs. We show that the same scaling results, as demonstrated in Table II, hold for PIL networks under $\mathrm{CoE}^{1-\mathrm{h}}-\mathrm{CoE}^{3-\mathrm{h}}$ (Theorem 3) and for IL networks under $\mathrm{CoE}^{1-\mathrm{h}}, \mathrm{CoE}^{2-\mathrm{h}}, \mathrm{CoE}^{4-\mathrm{h}}$ and $\mathrm{CoE}^{5-\mathrm{h}}$ (Theorem 4) even for such more heterogeneous CoEs. For IL networks under $\mathrm{CoE}^{3-\mathrm{h}}$, we show that the sum-rate capacity still scales logarithmically with $N$, and obtain upper and lower bounds for the pre-log factor in this scaling. (See Theorem 4 for more details.)

\section{A Note on Notation and Paper Organization}

When we write $p(x)=O(q(x))$ and $p(x)=o(q(x))$ for two positive functions $p(x)$ and $q(x)$, we mean $\limsup _{x \rightarrow \infty} \frac{p(x)}{q(x)}<\infty$ and $\lim _{x \rightarrow \infty} \frac{p(x)}{q(x)}=0$, respectively.

As is standard in the literature [31], a wireless channel is said to be a Rayleigh fading channel if the channel magnitude gain is Rayleigh distributed, or equivalently the channel power gain is exponentially distributed. A wireless channel is said to be a Rician- $K$ fading channel if the channel magnitude gain is Rician distributed with a Rician factor $K$. For a Rician- $K$ fading channel, the channel power gain is non-central chi-square distributed with two degrees of freedom. A wireless channel is said to be a Nakagami- $m$ fading channel if the channel magnitude gain is Nakagami distributed with a Nakagami factor $m \geq 0.5$. For a Nakagami- $m$ fading channel, the channel power gain is Gamma distributed. We refer the reader to [31] for more details about fading distributions.

In what follows, when we say a $\mathrm{CoE}$ is homogeneous, we mean that the parameters of the fading models are the same across the SUs, and all STSB and STPB channels have unit mean power gain. $\mathrm{CoE}^{i}$ notation is used to denote the $i$ th homogeneous $\mathrm{CoE}$. When we say a $\mathrm{CoE}$ is heterogeneous, we mean that fading parameters for different SUs are different. For heterogeneous CoEs , $K_{i}$ and $m_{i}$ are used to represent the Rician $K$ factor and Nakagami $m$ factor for the $i$ th SU, respectively, while $\alpha_{i}$ is used to represent the mean channel power gain for the $i$ th SU for Rayleigh fading channels. The mean channel power gain for Rician and Nakagami fading channels is denoted by $\Omega_{i}$. $\mathrm{CoE}^{i-\mathrm{h}}$ notation is used to denote the $i$ th heterogeneous CoE.

We consider five different communication scenarios (CoS) each for the homogeneous and the heterogeneous cases, as illustrated in Table I. Although Rayleigh fading can be obtained as a special case of Nakagami- $m(m=1)$ or Rician- $K(K=0)$, we have explicitly left the Rayleigh-Rayleigh combination as a separate entry since this is the most popular communication scenario usually investigated. Note also that due to the lack of closed-form expressions of the distribution function of the "joint power and interference channel state" for the CoSs involving Nakagami-Nakagami, Rician-Rician, Rician-Nakagami, and Nakagami-Rician fading, we have not included these scenarios in Table I. Derivation of throughput scaling laws for these CoSs will be left for future work. For the homogeneous case, the CoSs in Table I will be referred to as $\mathrm{CoS}_{\mathrm{PIL}}^{i}$ and $\operatorname{CoS}_{\mathrm{IL}}^{i}$ for $i \in\{1,2, \ldots, 5\}$ for PIL and IL networks, respectively. For the heterogeneous case, they will be referred to as $\operatorname{CoS}_{\mathrm{PIL}}^{i-\mathrm{h}}$ and $\operatorname{CoS}_{\mathrm{IL}}^{i-\mathrm{h}}$ for $i \in\{1,2, \ldots, 5\}$ for PIL and IL networks, respectively. We will use $R_{\mathrm{PIL}}^{i}(N)$ to denote the sum-rate in $\operatorname{CoS}_{\mathrm{PIL}}^{i}$ with $N$ SUs, which represents the sum-rate for a PIL network consisting of $N$ SUs with statistical channel models given as in $\mathrm{CoE}^{i}$. Similarly, $R_{\mathrm{IL}}^{i}(N), R_{\mathrm{PIL}}^{i-\mathrm{h}}(N)$ and $R_{\mathrm{IL}}^{i-\mathrm{h}}(N)$ denote the sum-rates in $\mathrm{CoS}_{\mathrm{IL}}^{i}, \mathrm{CoS}_{\mathrm{PIL}}^{i-\mathrm{h}}$ and $\mathrm{CoS}_{\mathrm{IL}}^{i-\mathrm{h}}$, respectively, when there are $N$ SUs in the secondary network.

The rest of the paper is organized as follows. In Section II, we compare and contrast our results with the relevant previous work. Section III describes the system model and network configuration along with our modeling assumptions. Section IV derives and presents the secondary network sum-rate scaling laws for different CoSs, provides various practical and theoretical insights into the derived sum-rate scaling laws, and illustrates the accuracy of our results, asymptotically correct in the large 
network limit with infinitely many SUs, by means of numerical analysis for cognitive radio networks with finitely many SUs. Section V concludes the paper. Our proofs are relegated to appendixes.

\section{RELATED WORK}

Our results in this paper are related to two main groups of works, which can be broadly classified under the names i) optimum resource/power allocation and ii) sum-rate capacity scaling via multiuser diversity, in the literature. Here, we will only mention the papers which are most relevant to ours.

The optimum allocation of transmission powers for primary networks is a well-understood research problem [12], [13], and [15]. On the other hand, optimum allocation of transmission powers in cognitive radio networks is a relatively new research area. The capacity achieving power allocation policy for a single SU under interference temperature constraints and the resulting data rates were obtained in [8]. In [16], Suraweera et al. considers the same setup in [8] and derive analogous results for asymmetric fading environments. Similar results were also extended to ergodic, delay-limited and outage capacities of cognitive radio networks with a single SU under various combinations of average and peak transmission and interference power constraints in [9]. Optimum power allocation policy achieving (ergodic) capacity was further obtained for multiple access and broadcast cognitive radio networks with multiple SUs under various combinations of average and peak transmission and interference power constraints in [10]. A survey on recent developments in the field can be found in [11].

Similar to these previous works, our analysis in this paper also starts with the consideration of sum-rate maximizing power allocation policy for multiple access cognitive networks under average total transmission power constraint and a constraint on the average interference caused by SUs to the primary network. Different from these works, our motivation here is to derive tight sum-rate scaling laws for cognitive radio networks with increasing numbers of SUs. This difference in the motivation leads us to consider a more stringent transmission power constraint than the ones considered in [9] and [10]. We show that, even with an average total power constraint on the secondary network, communication rates growing large are attainable with increasing numbers of SUs thanks to MDGs.

Capacity scaling laws and multiuser diversity gains are extensively studied for primary networks, e.g., see [19]-[21]. However, these works are only limited to Rayleigh fading channels. On the other hand, we study more general fading channels such as Rician and Nakagami- $m$ fading channels, which include the Rayleigh fading model as a special case, in this paper. Even though our problem formulation involving multiple access channel with average transmission and interference power constraints is much different than those studied in [19]-[21], similar techniques [22] are used to derive capacity scaling laws under both formulations. Therefore, some parts of our analysis are expected to find greater applicability to extend MDGs obtained for the dual broadcast channels in primary networks beyond Rayleigh fading communication environments.

Related work also includes multiuser and multispectrum diversity results in cognitive radio networks [23]-[26]. In [23], they performed a joint investigation of multiuser and multispectrum diversity gains for a cognitive radio network consisting of a broadcast secondary network and a primary network with multiple orthogonal frequency bands. For Rayleigh fading channels, they derived analytical expressions characterizing the secondary network capacity when PUs are protected by a peak interference power constraint. For a network model similar to the one studied in [23], the authors in [24] considered secondary network sum-rate capacity scaling under optimum matching of $M$ SUs with $M$ primary network frequency bands. Without considering any protection on the primary network, they obtained a double-logarithmic scaling law for the secondary network capacity for Rayleigh fading channels. Scaling laws for the secondary network capacity under simultaneous peak transmission power and peak interference power constraints have also been obtained recently in [25] and [26]. The authors in [25] studied the capacity scaling laws for a multiple access secondary network for Rayleigh fading channels, while [26] extends these results to multiple access, broadcast and parallel access secondary networks. Similar to our results here, these papers establish logarithmic and double-logarithmic secondary network capacity scaling behavior for peak transmission power and peak interference power limited cognitive radio networks under some approximations. Finally, capacity scaling laws of a cognitive network with non-fading channels but with a random distribution of cognitive users have been established in [27], whereas throughput scaling laws with spatially Poisson distributed primary users and spatially Poisson distributed secondary users have been investigated in [28] in the sense of the seminal result by Gupta and Kumar [29].

The current paper differs from the above-mentioned recent work on multiuser and multispectrum diversity in cognitive radio networks on several important fronts. First of all, we consider average (total) transmission power constraints on the secondary network and average interference power protection for the primary network, as opposed to peak transmission power and peak interference power constraints in all previous work. From a practical point of view, average transmission and interference power constraints are more suitable for delay insensitive traffic. From a technical point of view, they lead to a different jointly optimum spectrum sharing and transmission power policy (see Lemma 1), which, in turn, leads to new estimates for the tail distribution of the received signal-to-interference-plus-noise ratio (SINR) at the secondary base station (SBS), e.g., see Appendix A and Appendix C. Second, we analyze secondary network capacity scaling for more general fading models including Rician and Nakagami- $m$ fading (see Tables I and II), whereas most of the previous work focused on Rayleigh fading channels for the purposes of analytical tractability. Finally, we consider more heterogeneous communication environments in which different SUs experience statistically different channel conditions (see Theorems 3 and 4), whereas all previous work assumed statistically identical power gains for SUs.

\section{SYSTEM MODEL}

Consider an underlay cognitive radio network in which $N$ SUs share a frequency band with a single PU as depicted in 


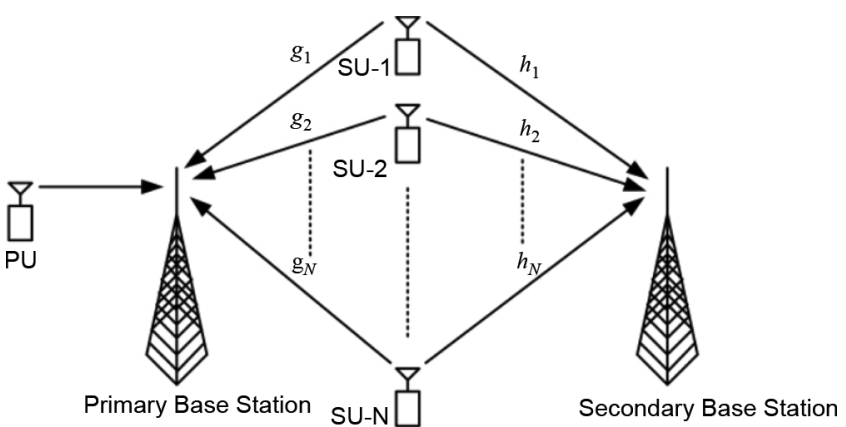

Fig. 1. $N$ SUs forming a multiple access channel to the SBS and interfering with signal reception at the PBS.

Fig. 1. Single PU assumption is just for the sake of clarity of final results, and various extensions to multiple PUs are possible as discussed at the end of this section. SUs form a multiple access channel to an SBS, and interfere with the signal reception at the primary base station (PBS). For $i \in\{1,2, \ldots, N\}$, we let $h_{i}$ be the $i$ th STSB channel power gain. Similarly, we let $g_{i}$ be the $i$ th STPB (interference) channel power gain. All channels are assumed to be ergodic block-fading channels with continuous power gain distributions as described in Table I. We assume the availability of full channel state information (CSI), i.e., availability of the random gain vectors $\boldsymbol{h}$ and $\boldsymbol{g}$ at the SBS.

We define a power allocation policy $\boldsymbol{P}(\boldsymbol{h}, \boldsymbol{g})=$ $\left(P_{1}(\boldsymbol{h}, \boldsymbol{g}), P_{2}(\boldsymbol{h}, \boldsymbol{g}), \ldots, P_{N}(\boldsymbol{h}, \boldsymbol{g})\right)^{\top}$ as a mapping from $\mathbb{R}_{+}{ }^{N} \times \mathbb{R}_{+}{ }^{N}$ to $\mathbb{R}_{+}{ }^{N}$, where $P_{i}(\boldsymbol{h}, \boldsymbol{g})$ represents the transmission power allocated to the $i$ th $\mathrm{SU}$ at the joint fading state $(\boldsymbol{h}, \boldsymbol{g})$. Let $\mathcal{P}$ be the space of all functions from $\mathbb{R}_{+}{ }^{N} \times \mathbb{R}_{+}{ }^{N}$ to $\mathbb{R}_{+}{ }^{N}$. We are interested in the solution of the following functional optimization problem [32]

$$
\begin{array}{cl}
\underset{\boldsymbol{P} \in \mathcal{P}}{\operatorname{maximize}} & \mathrm{E}_{\boldsymbol{h}, \boldsymbol{g}}\left[\log \left(1+\boldsymbol{h}^{\top} \boldsymbol{P}(\boldsymbol{h}, \boldsymbol{g})\right)\right] \\
\text { subject to } & \mathrm{E}_{\boldsymbol{h}, \boldsymbol{g}}\left[\mathbf{1}^{\top} \boldsymbol{P}(\boldsymbol{h}, \boldsymbol{g})\right] \leq P_{\text {ave }} \\
& \mathrm{E}_{\boldsymbol{h}, \boldsymbol{g}}\left[\boldsymbol{g}^{\top} \boldsymbol{P}(\boldsymbol{h}, \boldsymbol{g})\right] \leq Q_{\text {ave }}
\end{array}
$$

where all expectations are taken over random vectors $\boldsymbol{h}$ and $\boldsymbol{g}$, $T$ represents the transpose operator, and $\mathbf{1}$ (in boldface) is the vector of ones. Note that, by taking the receiver noise variance at the SBS as unity without loss of generality, our objective function for a given power allocation policy $\boldsymbol{P}$ represents the multiple access sum-rate capacity under $\boldsymbol{P}$, which can be achieved by using complex Gaussian codebooks and successive signal decoding at the SBS [13].

Remark 1: Similar to [8] and [10], we assume that the effect of primary's interference on the throughput of secondary network is negligible. This assumption is valid when either the primary's interference at the secondary receiver is not significant or the secondary user's receiver can decode the primary's data (if the primary has a strong signal for example) and cancel the resulting interference.

For the sake of simplicity, we will start our analysis by considering homogeneous CoEs in which all STSB gains are independent and identically distributed (i.i.d.) as well as STPB gains. That is, all $h_{i}$ 's and $g_{i}$ 's are i.i.d. across SUs, and the random vectors $\boldsymbol{h}=\left(h_{1}, h_{2}, \ldots, h_{N}\right)^{\top}$ and $\boldsymbol{g}=\left(g_{1}, g_{2}, \ldots, g_{N}\right)^{\top}$ are also independent. We will relax this assumption later. The next lemma gives the optimal power control policy $\boldsymbol{P}^{\star}$ solving (1). Since $P^{\star}$ is in the form of a water-filling policy, it is, indeed, the jointly optimum spectrum sharing and power control policy instructing us how to share the common spectrum among SUs and the PU, and to perform power control optimally. We will skip its proof since similar proofs have already appeared in [10] under various combinations of average and peak transmission power and interference power constraints.

Lemma 1: $\boldsymbol{P}^{\star}$ is given by the following water-filling scheme:

$$
\begin{aligned}
& P_{i}^{\star}(\boldsymbol{h}, \boldsymbol{g}) \\
& \quad= \begin{cases}\left(\frac{1}{\lambda_{N}+\mu_{N} g_{i}}-\frac{1}{h_{i}}\right)^{+}, & \text {if } \frac{h_{i}}{\lambda_{N}+\mu_{N} g_{i}} \geq \frac{h_{j}}{\lambda_{N}+\mu_{N} g_{j}}, \forall j \neq i \\
0, & \text { otherwise }\end{cases}
\end{aligned}
$$

where $(x)^{+}=\max (x, 0), \lambda_{N} \geq 0$ is the Lagrange multiplier associated with the average total transmission power constraint in (1) and $\mu_{N} \geq 0$ is the Lagrange multiplier associated with the average interference power constraint in (1).

Note that we have used $\lambda_{N}-\mu_{N}$ notation, rather than the more common $\lambda-\mu$ notation, to show the explicit dependence of Lagrange multipliers on $N$ in Lemma 1 . We define $X_{i, N}$ and $X_{N}^{\star}$ as $X_{i, N}=\frac{h_{i}}{\lambda_{N}+\mu_{N} g_{i}}$ and $X_{N}^{\star}=\max _{1 \leq i \leq N} X_{i, N}$. Here, $X_{i, N}$ can be interpreted as the joint power and interference channel state of the $i$ th SU. Then, under $\boldsymbol{P}^{\star}$, we schedule the $i$ th SU for transmission with power $\left(\frac{1}{\lambda_{N}+\mu_{N} g_{i}}-\frac{1}{h_{i}}\right)^{+}$if and only if the $i$ th SU has the best joint power and interference channel state, i.e., $X_{i, N}=X_{N}^{\star}$. Therefore, the sum-rate achieved by $\boldsymbol{P}^{\star}$ is given by $R_{N}=\mathrm{E}\left[\log \left(X_{N}^{\star}\right) 1_{\left\{X_{N}^{\star} \geq 1\right\}}\right]$.

The primary purpose of this paper is to identify the scaling behavior of $R_{N}$ under various CoSs as the number of SUs becomes large. To this end, we analyze two different network types, first of which is PIL networks in which the network operation is limited by both an average total power constraint on the secondary network and an average interference constraint on the primary network. Technically speaking, we have $\lambda_{N}>0$ and $\mu_{N}>0$ for this network type. The second network type is IL networks in which the network operation is only limited by an average interference constraint on the primary network. Technically speaking, we have $\lambda_{N}=0$ and $\mu_{N}>0$ for this network type.

Various extensions of our results to cognitive radio networks consisting of multiple PUs are possible. For example, if PUs also form a multiple access channel to a PBS with an average power constraint, the optimum power allocation strategy maximizing their sum-rate is to schedule only one PU with the best CSI for transmission according to a water-filling power control scheme [12], [13]. Therefore, SUs effectively need to protect transmissions only from a single PU at each channel use, and most of our results can be extended to this case straightforwardly. More generally, we can consider a primary network in which $M$ different PUs are scheduled for communication with $M$ different primary receivers. In this case, it can be shown that the $i$ th $\mathrm{SU}$ is scheduled for transmission (to maximize secondary network sum-rate) if and only if $\frac{h_{i}}{\lambda_{N}+\sum_{k=1}^{M} \mu_{k, N} g_{i, k}} \geq$ $\frac{h_{j}}{\lambda_{N}+\sum_{k=1}^{M} \mu_{k, N} g_{j, k}}$ for all $j \in\{1,2, \ldots, N\}$, where $g_{i, k}$ is the 


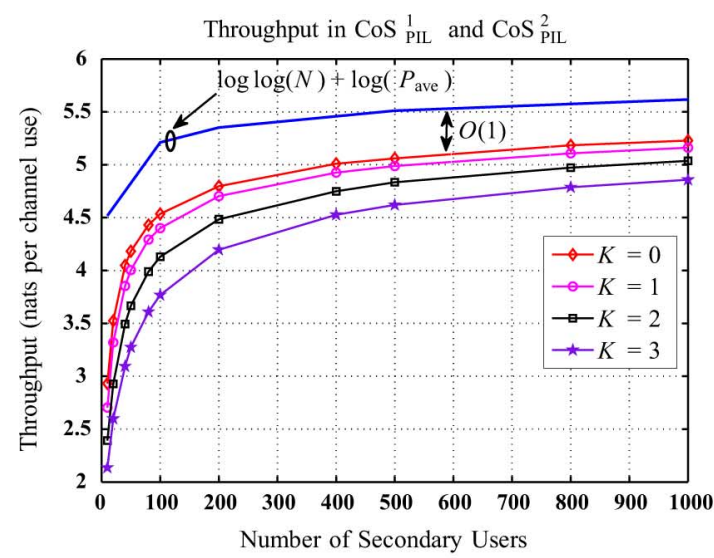

(a)

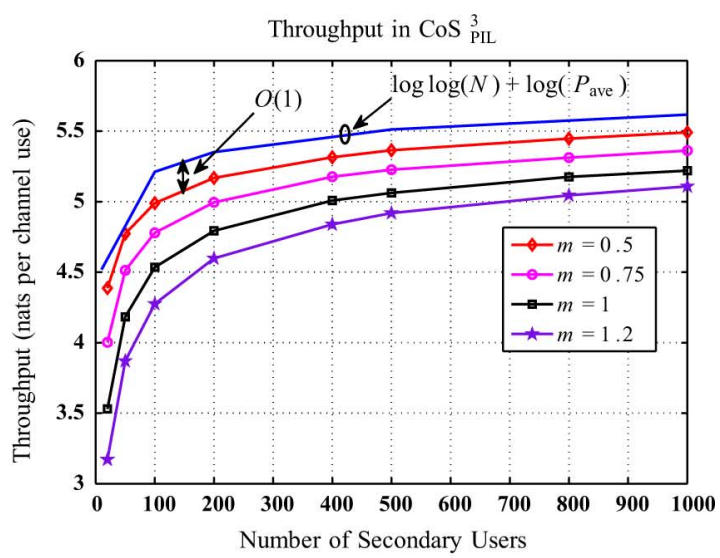

(b)

Fig. 2. Throughput scaling in $\operatorname{CoS}_{\mathrm{PIL}}^{i}, i \in\{1,2,3\}$, with $P_{\text {ave }}$ and $Q_{\text {ave }}$ set to 16 and $0 \mathrm{~dB}$, respectively.

interference channel power gain between the $i$ th $\mathrm{SU}$ and the $k$ th primary receiver, $\lambda_{N}$ is the Lagrange multiplier associated with the average total power constraint (on the secondary network), and $\mu_{k, N}$ is the Lagrange multiplier associated with the average interference power constraint at the $k$ th primary receiver. If all $\mu_{k, N} \mathrm{~S}$ are the same and equal to $\mu_{N}$ and all channels are i.i.d. Rayleigh fading channels, then $\mu_{N} \sum_{k=1}^{M} g_{i, k}$ becomes a Gamma distributed random variable. Therefore, exactly the same throughput scaling result obtained for $\mathrm{CoS}_{\mathrm{PIL}}^{3}$ continues to hold for this multiple PUs case. Furthermore, even if interference channels between SUs and primary receivers are i.i.d. Nakagami- $m$ fading channels, a similar discussion shows that the same throughput scaling still holds since Gamma distributions form a stable family of distributions.

\section{MAin Results AND Discussions}

In this section, we state the main asymptotic sum-rate scaling results of the paper along with numerical analysis illustrating them for finite networks.

\section{A. Scaling Results for Homogeneous CoEs}

We start our discussion by establishing the sum-rate scaling laws in homogeneous CoEs. Later, we will build upon these results to derive the sum-rate scaling laws in heterogeneous CoEs. The next theorem states the sum-rate scaling results in $\operatorname{CoS}_{\mathrm{PIL}}^{1}-\mathrm{CoS}_{\mathrm{PIL}}^{3}$.

Theorem 1: The sum-rate $R_{\mathrm{PIL}}^{i}(N)$ in $\mathrm{CoS}_{\mathrm{PIL}}^{i}$ scales according to $\lim _{N \rightarrow \infty} \frac{R_{\mathrm{PL}}^{i}(N)}{\log \log (N)}=1$ for $i=1,2$, and 3 .

Proof: Please refer to Appendix A.

In Appendix A, we give a detailed proof of Theorem 1 for $\operatorname{CoS}_{\text {PIL }}^{1}$ in which STSB channels are Rayleigh fading channels and STPB channels are Rician- $K$ fading channels. $\operatorname{CoS}_{\mathrm{PIL}}^{2}$ can be obtained as a special case of $\operatorname{CoS}_{\text {PIL }}^{1}$ by setting $K$ to 0 , and only key proof ideas for $\operatorname{CoS}_{\mathrm{PIL}}^{3}$ are illustrated to avoid repetitions.

Theorem 1 formally establishes $\log \log (N)$-type scaling behavior for the sum-rate in $\mathrm{CoS}_{\mathrm{PIL}}^{1}-\mathrm{CoS}_{\mathrm{PIL}}^{3}$. Furthermore, based on our analysis in Appendix A, we can also characterize the destructive effect of Rician fading on the sum-rate scaling in
$\operatorname{CoS}_{\mathrm{PIL}}^{1}$ for finite numbers of SUs. That is, $R_{\mathrm{PIL}}^{1}(N)$ can be lower and upper bounded as

$$
\begin{aligned}
\log \log \left(\frac{K+1}{\mathrm{e}^{K}} N\right)+O(1) & \leq R_{\mathrm{PIL}}^{1}(N) \\
& \leq \log \log \left(\frac{K+1}{\mathrm{e}^{K}} N\right)+O(1)
\end{aligned}
$$

by using (8), (11), and (12). This result suggests that the destructive effect of Rician fading on the throughput in $\mathrm{CoS}_{\mathrm{PIL}}^{1}$ can be regarded as reducing the number of SUs by a factor of $\frac{K+1}{\mathrm{e}^{K}}$. Based on this observation, we define the effective number of users contributing to MDG in $\operatorname{CoS}_{\mathrm{PIL}}^{1}$ as $\frac{K+1}{\mathrm{e}^{K}} N$. Note that $\frac{K+1}{\mathrm{e}^{K}}$ is a decreasing function of $K, \frac{K+1}{\mathrm{e}^{K}} \leq 1$ and the equality is achieved when $K=0$. This implies that the effective number of SUs is maximum when $K=0$ (i.e., no line-of-sight component between SUs and the PBS), and decreases with increasing $K$. Since increasing $K$ means more power in the line-of-sight fading components, this finding quantifies the effect of line-of-sight fading components between SUs and the PBS on the secondary network data rates. Finally, we establish an important convergence property for Lagrange multipliers $\lambda_{N}$ in Appendix B by showing that $\lambda_{N}$ converges to $\frac{1}{P_{\text {ave }}}$ as $N$ increases in all CoSs for PIL networks. This result indicates that $R_{\mathrm{PIL}}^{i}(N), i \in\{1,2,3\}$, is almost the same with the data rates calculated by fixing $\lambda_{N}$ at $\frac{1}{P_{\text {ave }}}$ in Appendix A for all $N$ large enough. Based on this observation and (8) and (11) in Appendix A, we conclude that an increase in $P_{\text {ave }}$ leads to a logarithmic increase on the secondary network throughput for PIL networks.

In Fig. 2, we demonstrate the throughput scaling behavior with increasing numbers of $\mathrm{SUs}$ in $\mathrm{CoS}_{\mathrm{PIL}}^{1}, \mathrm{CoS}_{\mathrm{PIL}}^{2}$ and $\mathrm{CoS}_{\mathrm{PIL}}^{3}$. In this figure, we set $P_{\text {ave }}$ to $16 \mathrm{~dB}$ and $Q_{\text {ave }}$ to $0 \mathrm{~dB}$. Similar qualitative behavior continues to hold for other values

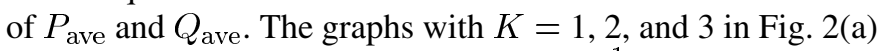
represent the throughput scaling in $\operatorname{CoS}_{\mathrm{PIL}}^{1}$ with different Rician factors, and the graph with $K=0$ in Fig. 2(a) represents the throughput scaling in $\mathrm{CoS}_{\mathrm{PIL}}^{2}$. Fig. 2(b) demonstrates the throughput scaling in $\operatorname{CoS}_{\mathrm{PIL}}^{3}$ for different values of Nakagami fading parameters. As Fig. 2 shows, an increase in the number of SUs leads to a corresponding increase in the secondary 
network throughput due to MDGs as predicted by Theorem 1 . In addition, the scaling behavior of the throughput, as shown in Fig. 2, is the same as the $\log \log (N)$-type of behavior in Theorem 1, up to a constant factor which comes from i) the effects of $P_{\text {ave }}$, and fading distribution parameters on the throughput of the secondary network, and ii) the higher order error terms due to the approximation process. In particular, the logarithmic effect of $P_{\text {ave }}$ on the throughput is further indicated by the closeness of the simulated data rates and the $\log \log (N)+\log \left(P_{\text {ave }}\right)$ curves in Fig. 2.

As the Rician factor $K$ in $\operatorname{CoS}_{\mathrm{PIL}}^{1}$ and the Nakagami fading parameter $m$ in $\mathrm{CoS}_{\mathrm{PIL}}^{3}$ increase, SUs' transmission powers become more limited due to more severe interference caused to the PBS, and as a result, we start to observe a decrease in the secondary network throughput. In $\operatorname{CoS}_{\mathrm{PIL}}^{1}$, the maximum throughput is achieved when there is no line-of-sight fading component between SUs and the PBS, i.e., $\operatorname{CoS}_{\mathrm{PIL}}^{2}$. Similarly, in $\operatorname{CoS}_{\mathrm{PIL}}^{3}$, the throughput is maximized with $m=0.5$, which corresponds to the mildest fading condition in STPB channels, as far as the SU throughput is concerned.

Now, we turn our attention to homogeneous IL networks. The next theorem establishes the secondary network throughput scaling behavior in $\operatorname{CoS}_{\mathrm{IL}}^{1}-\operatorname{CoS}_{\mathrm{IL}}^{5}$. In Appendix $\mathrm{C}$, we give a detailed proof of Theorem 2 for $\operatorname{CoS}_{\mathrm{IL}}^{1}$ in which STSB channels are Rayleigh fading channels, and STPB channels are Rician- $K$ fading channels. $\operatorname{CoS} \mathrm{S}_{\mathrm{IL}}^{2}$ can be obtained as a special case of $\operatorname{CoS}_{\mathrm{IL}}^{1}$ by setting $K$ to 0 , and only key proof ideas for other CoSs are illustrated to avoid repetitions.

Theorem 2: The sum-rate $R_{\mathrm{IL}}^{i}(N)$ in $\operatorname{CoS}_{\mathrm{IL}}^{i}$ scales according to $\lim _{N \rightarrow \infty} \frac{R_{\mathrm{IL}}^{i}(N)}{\log (N)}=1$ for $i \in\{1,2,4,5\}$ and $\lim _{N \rightarrow \infty} \frac{R_{\mathrm{IL}}^{i}(N)}{\log (N)}=\frac{1}{m}$ for $i=3$.

Proof: Please refer to Appendix C.

We will start our discussion on the secondary network throughput scaling for IL networks by first analyzing the effect of various system parameters on the throughput scaling and providing effective number of users interpretations. Then, we will compare and contrast our analytical throughput scaling results with our numerical results obtained through simulations for finite networks.

The fundamental distinction between the throughput scaling results for IL networks and those established for PIL networks is that the secondary network throughput now scales according to $\log (N)$, whereas this scaling behavior was $\log \log (N)$ in previously studied CoSs for PIL networks. Therefore, by relaxing the power constraint on the secondary network, we achieve an exponential gain in secondary network data rates. Furthermore, we can upper and lower bound $R_{\mathrm{IL}}^{1}(N)$ as (2), shown at the bottom of the page, by using (20), (22), and (23). This implies that the effective number of users contributing to MDGs in $\operatorname{CoS}_{\mathrm{IL}}^{1}$ is given by $\frac{K+1}{\mathrm{e}^{K}} N$, which is the same effective number of users given for $\operatorname{CoS}_{\mathrm{PIL}}^{1}$. In $\operatorname{CoS}_{\mathrm{IL}}^{3}$, we can lower and upper bound $R_{\mathrm{IL}}^{3}(N)$ as (3), shown at the bottom of the page, by using (26) and (27). This finding implies an effective number of users of $m N^{\frac{1}{m}}$ contributing to MDGs in $\operatorname{CoS}_{\mathrm{IL}}^{3}$. In addition, effective numbers of users in $\operatorname{CoS}_{\mathrm{IL}}^{4}$ and $\operatorname{CoS}_{\mathrm{IL}}^{5}$ can be given by $N$ (i.e., see (29), (30), (34), and (35)), implying that MDGs in these CoSs do not critically depend on the STSB channel fading parameters. Finally, we establish an important asymptotic behavior of Lagrange multipliers $\mu_{N}$ in Appendix D by showing that $\mu_{N}$ converges to $\frac{1}{Q_{\text {ave }}}$ as $N$ tends to infinity in all CoSs for IL networks. Based on this observation and (20), (22), (26), (29), and (34) in Appendix C, we conclude that an increase in $Q_{\text {ave }}$ leads to a logarithmic increase on the secondary network throughput for IL networks.

Although our main focus in this paper is to consider an average total transmission power constraint across the SUs, it is also possible to envisage other scenarios in which all SUs have their own individual average transmission power constraints. In these cases, each SU brings its individual power resources in, and having more SUs implies higher average total transmission power available for the secondary network. Intuitively, this suggests that a secondary network with individual power constraints becomes an IL network as the number of SUs tends to infinity. This idea can be made rigorous by assuming that all SUs have the same average transmission power constraint $P_{\text {ave }}$ and by showing that (identical) Lagrange multipliers $\lambda_{N}$ associated with the individual transmission power constraints must scale according to $\lambda_{N}=o(1)$ in these cases. The proof of this result is not difficult, and essentially it follows by showing that $\lambda_{N} \leq \frac{1}{N P_{\text {ave }}}$. Details are omitted due to space restrictions. From a theoretical point of view, such a scaling behavior of $\lambda_{N}$ suggests that as $N$ becomes large, transmission powers of SUs are mainly limited by the average interference constraint rather than the individual power constraints. Therefore, throughput scaling behavior of a secondary network with individual power constraints will be similar to an IL network, i.e., we observe logarithmic growth in the rate with the number of SUs. A rigorous proof of this result for all possible CoSs is not provided here due to space constraints, but as an example, we provide a sketch of the proof of this result for $\mathrm{CoE}^{2}$ in the next lemma.

$$
\log \left(\frac{K+1}{\mathrm{e}^{K}} N\right)\left(1-O\left(\frac{\log \log (N)}{\log (N)}\right)\right)+O(1) \leq R_{\mathrm{IL}}^{1}(N) \leq \log \left(\frac{K+1}{\mathrm{e}^{K}} N\right)\left(1+O\left(\frac{\log \log (N)}{\log (N)}\right)\right)+O(1)
$$

$$
\log \left(m N^{\frac{1}{m}}\right)\left(1-O\left(\frac{\log \log (N)}{\log (N)}\right)\right)+O(1) \leq R_{\mathrm{IL}}^{3}(N) \leq \log \left(m N^{\frac{1}{m}}\right)\left(1+O\left(\frac{\log \log (N)}{\log (N)}\right)\right)+O(1)
$$




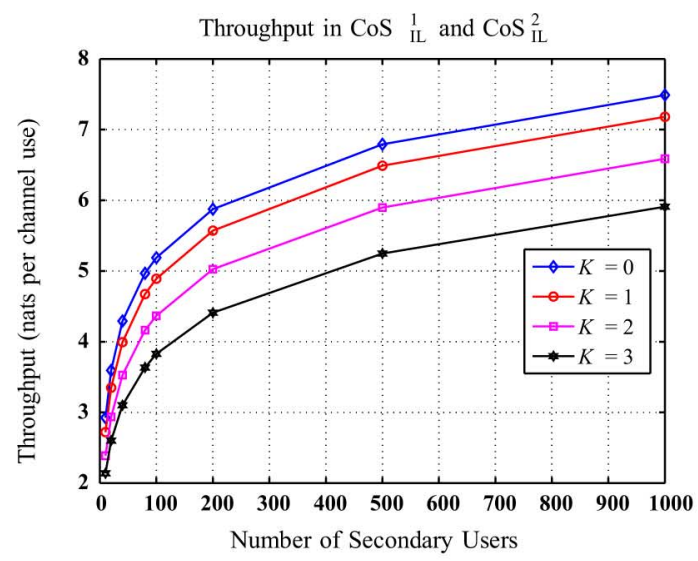

(a)

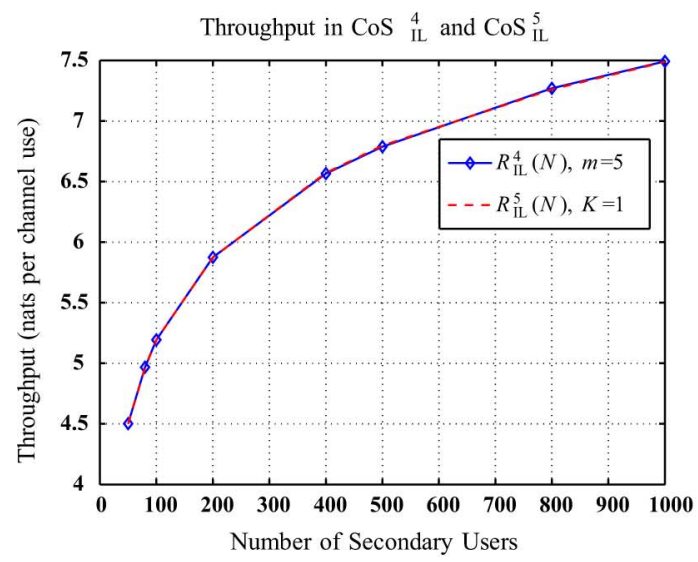

(c)

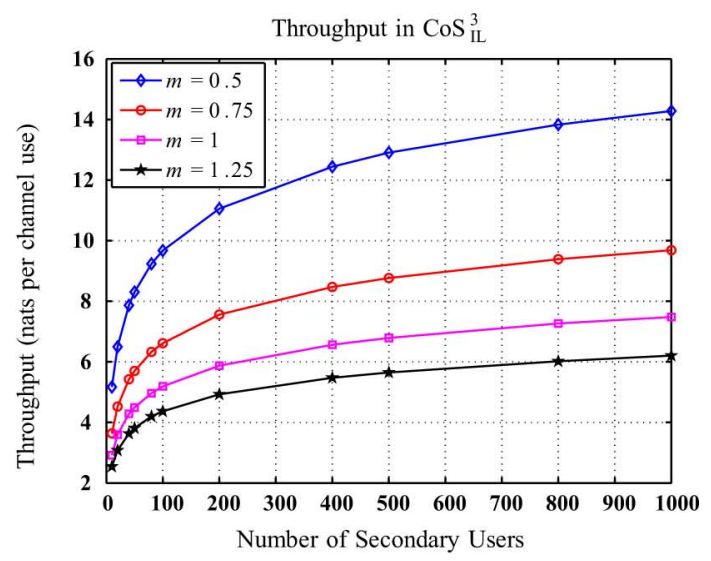

(b)
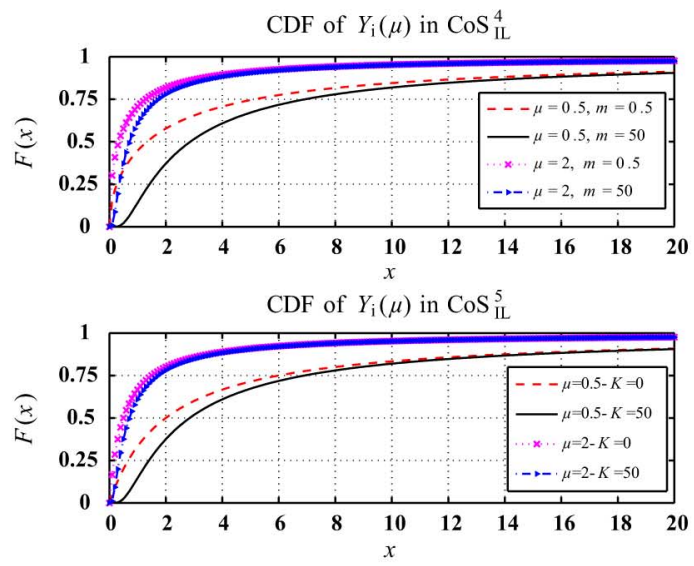

(d)

Fig. 3. Throughput scaling in $\operatorname{CoS}_{\mathrm{IL}}^{i}, i \in\{1,2, \ldots, 5\}$, with $Q_{\text {ave }}$ set to $0 \mathrm{~dB}((\mathrm{a})-(\mathrm{c}))$. The $\operatorname{CDF}$ of $Y_{i}(\mu)=\frac{h_{i}}{\mu g_{i}}$ for $\operatorname{CoS}_{\mathrm{IL}}^{4}$ and $\operatorname{CoS}_{\mathrm{IL}}^{5}(\mathrm{~d})$.

Lemma 2: Consider a secondary network with a symmetric individual average transmission power constraint of $P_{\text {ave }}$ and an average interference constraint $Q_{\text {ave }}$. Let $R(N)$ be the ergodic sum-rate under $\mathrm{CoE}^{2}$ in this case, with the optimal $\mathrm{SU}$ transmission power allocation policy (derived in [10]). Then, $\lim _{N \rightarrow \infty} \frac{R(N)}{\log (N)}=1$.

Proof: Note that the throughput in this network is upper bounded by an IL network under $\mathrm{CoE}^{2}$ with the same average interference constraint. Thus, $\lim \sup _{N \rightarrow \infty} \frac{R(N)}{\log (N)} \leq 1$. To show the other way, consider a suboptimal power allocation policy, $\boldsymbol{P}$, in which the power of the $i$ th $\mathrm{SU}$ is given by $P_{i}=$ $\alpha N 1_{\left\{g_{i}=g_{\min }\right\}}$, where $\alpha \in\left(0, \min \left(P_{\text {ave }}, Q_{\text {ave }}\right)\right]$ and $g_{\min }=$ $\min _{1 \leq i<N} g_{i}$. Let $R_{\boldsymbol{P}}(N)$ be the throughput of the secondary network when the transmission powers of SUs are allocated according to $\boldsymbol{P}$. Then, it follows that $R_{\boldsymbol{P}}(N) \leq R(N)$ and $\lim _{N \rightarrow \infty} \frac{{ }^{R} P^{(N)}}{\log (N)}=1$, which implies $\liminf _{N \rightarrow \infty} \frac{R(N)}{\log (N)} \geq$ 1 . This completes the proof.

Remark 2: Operating in an IL scenario does not necessarily imply that the average total transmission power of the secondary network is infinite. It can be shown that the average transmission power in $\operatorname{CoS}_{\mathrm{IL}}^{3}$ for $m>1$ is finite while the network is IL.

We now present our numerical results for throughput scaling in IL networks. In Fig. 3(a), we depict the simulated secondary network throughput for $\operatorname{CoS}_{\mathrm{IL}}^{1}$ and $\operatorname{CoS} \mathrm{IL}_{\mathrm{IL}}^{2}$ with $Q_{\text {ave }}$ fixed at 0 $\mathrm{dB}$. For other values of $Q_{\text {ave }}$, similar qualitative behavior con- tinues to hold. The graphs with $K=1,2$, and 3 in Fig. 3(a) represent the throughput scaling in $\mathrm{CoS}_{\mathrm{IL}}^{1}$ with different Rician factors, and the graph with $K=0$ in Fig. 3(a) represents the throughput scaling in $\operatorname{CoS}_{\mathrm{IL}}^{2}$. As shown in Fig. 3(a), throughput increases logarithmically with the number of SUs, which is consistent with the MDGs predicted by Theorem 2. As the power in line-of-sight components of Rician fading STPB channels increases, the interference caused to the PBS by the secondary network becomes more severe. To compensate this effect, SUs reduce their transmission power, which results in a throughput reduction in the secondary network as illustrated in Fig. 3(a).

In Fig. 3(b), we plot the secondary network throughput in $\mathrm{CoS}_{\mathrm{IL}}^{3}$ for $m=0.5,0.75,1$, and 1.25 with $Q_{\text {ave }}$ fixed at $0 \mathrm{~dB}$. Theorem 2 predicts an $\frac{1}{m} \log (N)$-type scaling behavior for the secondary network throughput, and our simulation results are in close agreement with this scaling behavior. For example, when the secondary network contains 1000 SUs, our simulation results give $6.2,7.5,9.7$, and 14.3 for secondary network data rates measured in nats per channel use for $m=1.25,1,0.75$, and 0.5 , respectively. On the other hand, data rates (in nats per channel use) predicted by Theorem 2 are 5.52, 6.9, 9.2, and 13.8 for $m=$ $1.25,1,0.75$, and 0.5 , respectively. As $m$ increases in Fig. 3(b), STPB channels become more deterministic, and we cannot utilize multiuser diversity effects in these (interference) channels to eliminate interference on the primary network. This leads to a decrease in SUs' transmission power levels to satisfy interfer- 


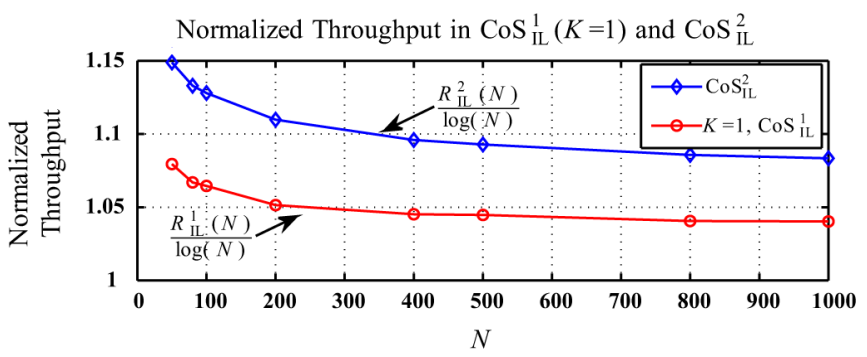

(a)

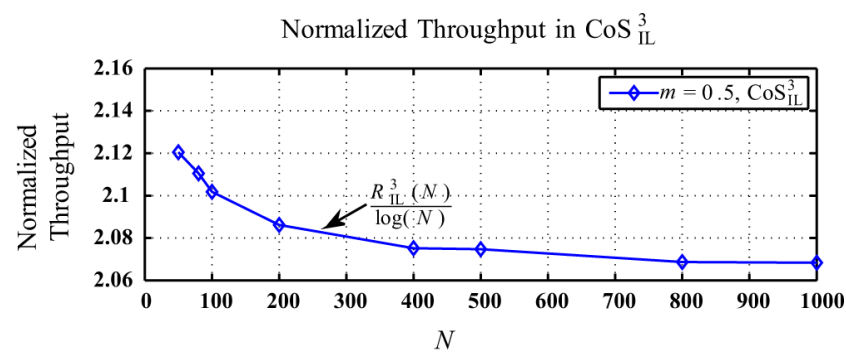

(b)

Fig. 4. Normalized secondary network throughput in $\operatorname{CoS}_{\mathrm{IL}}^{1}(K=1), \operatorname{CoS}_{\mathrm{IL}}^{2}$ and $\operatorname{CoS}_{\mathrm{IL}}^{3}(m=0.5)$. $Q_{\text {ave }}$ is fixed in $0 \mathrm{~dB}$.

ence constraints, and finally we observe an associated drop in the secondary network throughput. It should also be noted that an increase in $m$ results in a more severe throughput reduction than the one observed in $\mathrm{CoS}_{\mathrm{IL}}^{1}$ with increasing $K$ due to an exponential dependence of effective number of users on $m$.

In Fig. 3(c), we plot the secondary network throughput in $\operatorname{CoS}_{\mathrm{IL}}^{4}$ and $\operatorname{CoS}_{\mathrm{IL}}^{5}$ for $m=5\left(\operatorname{CoS}_{\mathrm{IL}}^{4}\right)$ and $K=1\left(\operatorname{CoS}_{\mathrm{IL}}^{5}\right)$ with $Q_{\text {ave }}$ fixed at $0 \mathrm{~dB}$. We observe very close data rates in both $\operatorname{CoS}_{\mathrm{IL}}^{4}$ and $\operatorname{CoS}_{\mathrm{IL}}^{5}$. In contrast to other CoSs, throughput scaling in $\operatorname{CoS}_{\mathrm{IL}}^{4}$ and $\operatorname{CoS}_{\mathrm{IL}}^{5}$ does not heavily depend on the fading parameters, and therefore we continue to observe similar throughput scaling behavior for other values of $m$ and $K$. In general, MDGs rely heavily upon the tail distributions of the underlying random variables of interest [34]. In our setup, such gains depend on the tail distribution of $X_{i, N}$. As illustrated in Appendix C and Lemma 11 in Appendix D, the tail distribution of $X_{i, N}$ can be approximated by the tail distribution of $Y_{i}(\mu)=\frac{h_{i}}{\mu g_{i}}$ with $\mu=\frac{1}{Q_{\text {ave }}}$ in $\operatorname{CoS}_{\mathrm{IL}}^{4}$ and $\operatorname{CoS}_{\mathrm{IL}}^{5}$ for $N$ large enough. In Fig. 3(d), we plot the CDF of $Y_{i}(\mu)$ for different values of $\mu, m$ and $K$ parameters. As observed in this figure, the tail distributions become indistinguishable in both CoSs for large values of $x$, which justifies why we observe very similar values for $R_{\mathrm{IL}}^{4}(N)$ and $R_{\mathrm{IL}}^{5}(N)$. Moreover, (29) and (34) suggest that throughput scaling in $\operatorname{CoS}_{\mathrm{IL}}^{4}$ and $\operatorname{CoS}_{\mathrm{IL}}^{5}$ does not depend on the fading parameters critically.

In Fig. 4, we plot the normalized secondary network throughput $\frac{R_{\mathrm{IL}}^{i}(N)}{\log (N)}$ as a function of the number of SUs for $\operatorname{CoS}_{\mathrm{IL}}^{1}-\operatorname{CoS}_{\mathrm{IL}}^{3}$ to further illustrate the accuracy of our scaling results. The normalized throughput $\frac{R_{\mathrm{L}}^{i}(N)}{\log (N)}$ converges to 1 for $\operatorname{CoS}_{\mathrm{IL}}^{1}$ and $\operatorname{CoS}_{\mathrm{IL}}^{2}$, whereas it converges to $\frac{1}{m}$ ( 2 in this case) for $\operatorname{CoS}_{\mathrm{IL}}^{3}$ with finite but large enough numbers of SUs, as predicted by Theorem 2 .

It is also instructive to study throughput scaling behavior of secondary networks as $K$ or $m$ tends to infinity. Consider first $\operatorname{CoS}_{\mathrm{PIL}}^{3}$ and $\operatorname{CoS}_{\mathrm{IL}}^{3}$. As $m$ tends to infinity in these CoSs, STPB channels become more and more deterministic, and signals transmitted from SUs are received by the PBS without any power degradation. Therefore, for large values of $m$, we cannot utilize multiuser diversity gains between SUs and the PBS, and the average interference constraint gradually reduces to a constraint on the transmission power. For $\mathrm{CoS}_{\mathrm{IL}}^{3}$, this implies that the network becomes a transmission power limited network with a maximum average permissible power level of $Q_{\text {ave }}$, and the throughput scaling gradually reduces from a $\log (N)$-type behavior to a $\log \log (N)$-type behavior.
In this case, it is possible to show that double logarithmic throughput gains are achieved by utilizing multiuser diversity gains between SUs and the SBS by following the same proof techniques used in this paper. Such a reduction in throughput is also consistent with our scaling results given in Theorem 2 for $\mathrm{CoS}_{\mathrm{IL}}^{3}$. However, Theorem 2 only predicts that $R_{\mathrm{IL}}^{3}(N)$ must be $o(\log (N))$ as $m$ tends to infinity without revealing precise throughput scaling dynamics. For $\mathrm{CoS}_{\mathrm{PIL}}^{3}$, the secondary network becomes a power limited network with a maximum average permissible power level of $\min \left(P_{\text {ave }}, Q_{\text {ave }}\right)$ as $m$ tends to infinity. $R_{\mathrm{PIL}}^{3}(N)$ still scales according to $\log \log (N)$, and hence the throughput scaling behavior in $\mathrm{CoS}_{\mathrm{PIL}}^{3}$ does not change with $m$. The same discussion also holds for $\operatorname{CoS}_{\mathrm{PIL}}^{1}$ and $\mathrm{CoS}_{\mathrm{IL}}^{1}$ as $K$ tends to infinity.

In Fig. 5(a), we plot the secondary network throughput in $\operatorname{CoS}_{\mathrm{PIL}}^{1}$ and $\operatorname{CoS}_{\mathrm{IL}}^{1}$ as a function of the Rician $K$ factor when the number of SUs is fixed either at $N=100$ or at $N=500$. We set $P_{\mathrm{ave}}=16 \mathrm{~dB}$ for $\operatorname{CoS}_{\mathrm{PIL}}^{1}$ and $Q_{\mathrm{ave}}=0 \mathrm{~dB}$ for $\operatorname{CoS}_{\mathrm{PIL}}^{1}$ and $\mathrm{CoS}_{\mathrm{IL}}^{1}$. For $N$ large enough, the secondary network throughput in $\operatorname{CoS}_{\mathrm{PIL}}^{1}$ and $\operatorname{CoS}_{\mathrm{IL}}^{1}$ can be approximated as $R_{\mathrm{PIL}}^{1}(N) \approx$ $\log \log (N)+\frac{\log \left(\frac{K+1}{K}\right)}{\log (N)}$ and $R_{\mathrm{IL}}^{1}(N) \approx \log (N)+\log \left(\frac{K+1}{\mathrm{e}^{K}}\right)$. This implies that the destructive effect of an upward change in $K$ on data rates is magnified by a factor of $\log (N)$ in $\operatorname{CoS}_{\mathrm{IL}}^{1}$ when compared to its effect on data rates in $\operatorname{CoS}_{\mathrm{PIL}}^{1}$. This is also confirmed through simulations in Fig. 5(a). As the power in the line-of-sight fading components increases, the secondary network throughputs in both network types decrease, but with a higher rate of change for the IL network. Some further qualitative insights are as follows. As $K$ increases, the IL network starts to behave more like a power limited network with an average power constraint $Q_{\text {ave }}$. On the other hand, as $K$ increases, the interference constraint becomes the more dominant constraint for the PIL network, and determines transmission power levels in $\operatorname{CoS}_{\mathrm{PIL}}^{1}$, i.e., average transmission power cannot exceed $Q_{\text {ave }}$. As a result, both network types essentially behave like power limited networks with the same power constraints, and the same data rates are achieved in both CoSs, as illustrated in Fig. 5(a). The same arguments continue to hold for data rates in $\operatorname{CoS}_{\mathrm{PIL}}^{3}$ and $\operatorname{CoS}_{\mathrm{IL}}^{3}$ as $m$ increases, as illustrated in Fig. 5(b).

\section{B. Scaling Results for Heterogeneous CoEs}

Here, we extend our secondary network throughput scaling results given for homogeneous CoEs above to heterogeneous CoEs in which different SUs are allowed to experience different channel conditions. To this end, we will let $\alpha_{i}$ represent the 


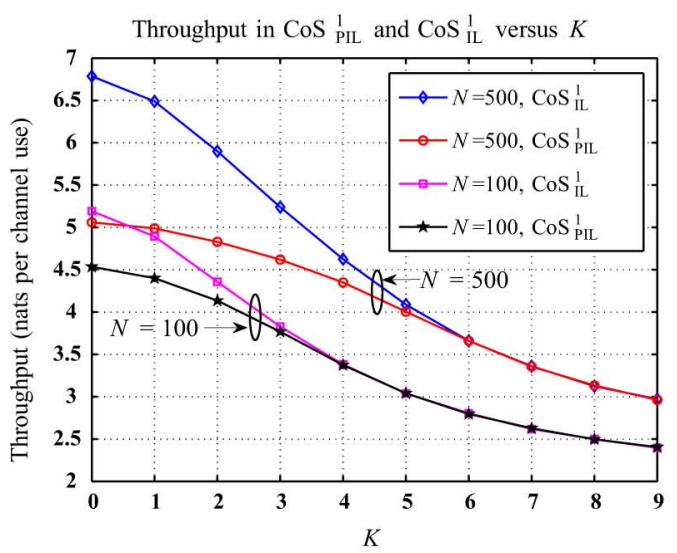

(a)

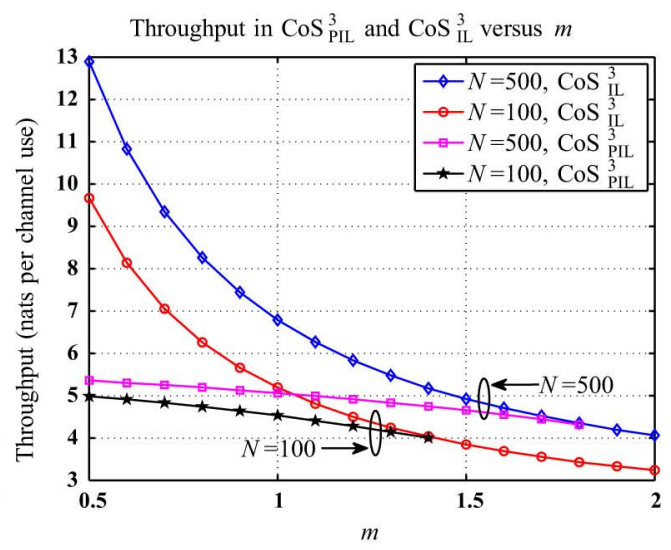

(b)

Fig. 5. Change of secondary network throughput (a) as a function of the Rician $K$ factor and (b) as a function of the Nakagami $m$ factor. (a) is given for CoS $S_{\mathrm{PIL}}^{1}$ and $\operatorname{CoS}_{\mathrm{IL}}^{1}$. (b) is given for $\operatorname{CoS}_{\mathrm{PIL}}^{3}$ and $\operatorname{CoS}_{\mathrm{IL}}^{3} \cdot N=100,500, P_{\text {ave }}=16 \mathrm{~dB}$ and $Q_{\text {ave }}=0 \mathrm{~dB}$.

mean channel power gain for the $i$ th Rayleigh fading channel, $\Omega_{i}$ represent the mean channel power gain for the $i$ th Rician or the $i$ th Nakagami fading channel, ${ }^{1} K_{i}$ represent the Rician factor for the $i$ th Rician fading channel, and $m_{i}$ represent the Nakagami parameter for the $i$ th Nakagami fading channel.

In a realistic scenario, it is expected that different SUs have different distances to the SBS and the PBS, and therefore have different mean channel power gains due to path-loss. It is also expected that different signals from different SUs are blocked by different obstacles existing in the network with various degrees of blocking, and therefore they have different channel power gains in the line-of-sight fading components. Our scaling results in this part are provided by taking all of these realistic factors into account.

We also need some technical assumptions in order to prove the throughput scaling results for the power-interference limited and the interference limited cases for the heterogeneous CoEs, formally presented below in Theorems 3 and 4, respectively. We let $\alpha_{\text {max }}=\sup _{i>1} \alpha_{i}, \alpha_{\text {min }}=\inf _{i \geq 1} \alpha_{i}, \Omega_{\max }=\sup _{i>1} \Omega_{i}$, $\Omega_{\text {min }}=\inf _{i \geq 1} \Omega_{i}, K_{\max }=\sup _{i>1} K_{i}, K_{\min }=\inf _{i \geq 1} K_{i}$, $m_{\text {max }}=\sup _{i \geq 1} m_{i}$ and $m_{\text {min }}=\inf _{i \geq 1} m_{i}$. It is assumed that $\alpha_{\min }>0, \Omega_{\min }>0, K_{\min } \geq 0, m_{\min }>0, \alpha_{\max }<\infty$, $\Omega_{\max }<\infty, K_{\max }<\infty$ and $m_{\max }<\infty$. These assumptions do not limit the generality of our scaling results below. By considering path-loss, $\alpha_{\min }>0, \Omega_{\min }>0, \alpha_{\max }<\infty$ and $\Omega_{\max }<\infty$ imply that SUs cannot be too close or too far away from the SBS and the PBS. $K_{\min } \geq 0$ and $m_{\min }>0$ are automatically satisfied by definition. When $K_{\max }=\infty$ or $m_{\max }=\infty$, the same calculations in Appendix E and $\mathrm{F}$ can be repeated for deterministic channels, and the same scaling results continue to hold for PIL networks, but the scaling results may change for IL networks from a $\log (N)$-type behavior to a $\log \log (N)$-type behavior as discussed above.

The next theorem establishes the throughput scaling behavior in $\operatorname{CoS}_{\mathrm{PIL}}^{1-\mathrm{h}}-\operatorname{CoS}_{\mathrm{PIL}}^{3-\mathrm{h}}$.

Theorem 3: The sum-rate $R_{\mathrm{PIL}}^{i-\mathrm{h}}(N)$ in $\mathrm{CoS}_{\mathrm{PIL}}^{i-h}$ scales according to $\lim _{N \rightarrow \infty} \frac{R_{\mathrm{PII}}^{i-\mathrm{h}}(N)}{\log \log (N)}=1$ for $i=1,2$, and 3 .

Proof: Please refer to Appendix E.

${ }^{1}$ Since Rician and Nakagami fading channel models do not appear together in the CoSs considered in this paper, this notation does not cause any confusion.

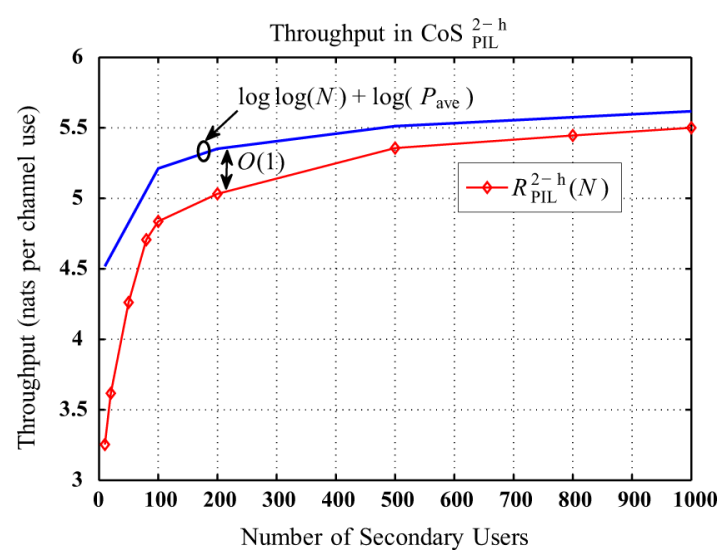

Fig. 6. Throughput scaling in $\operatorname{CoS}_{\mathrm{PIL}}^{2-\mathrm{h}}$ with $P_{\text {ave }}$ and $Q_{\text {ave }}$ set to $16 \mathrm{~dB}$ and 0 $\mathrm{dB}$, respectively. $\alpha_{i}$ 's are uniformly distributed over $[0.2,1.8]$.

In Appendix E, we only give the proof of this theorem for $\mathrm{CoS}_{\mathrm{PIL}}^{1-\mathrm{h}}$ and $\mathrm{CoS}_{\mathrm{PIL}}^{3-\mathrm{h}} \cdot \mathrm{CoS}_{\mathrm{PIL}}^{2-\mathrm{h}}$ is a special case of $\mathrm{CoS}_{\mathrm{PIL}}^{1-\mathrm{h}}$, and is obtained by setting Rician factors to zero. Note also that the coexistence of SUs experiencing Rayleigh fading and Rician fading STPB channels is allowed in $\mathrm{CoS}_{\mathrm{PIL}}^{1-\mathrm{h}}$ in its full generality. Theorem 3 demonstrates that the $\log \log (N)$-type throughput scaling behavior in PIL networks is not changed even if different SUs experience different fading channel conditions.

In Fig. 6, we demonstrate the sum-rate of $\operatorname{CoS}_{\mathrm{PIL}}^{2-\mathrm{h}}$ with increasing numbers of SUs. In this figure, we set $P_{\text {ave }}$ to $16 \mathrm{~dB}$ and $Q_{\text {ave }}$ to $0 \mathrm{~dB}$. We also assume that the means of STSB and STPB channel gains are uniformly distributed over $[0.2,1.8]$. In these simulations, the sum-rate for each $N$ is averaged over 100000 independent channel realizations with a fixed set of mean channel gains generated uniformly at random over $[0.2,1.8]$, as mentioned above. As $N$ increases, the mean channel gains of the existing users are kept fixed, whereas the mean channel gains of the new users are chosen again randomly from the uniform distribution over [0.2,1.8]. As Fig. 6 shows, the sum-rate in $\operatorname{CoS}_{\mathrm{PIL}}^{2-\mathrm{h}}$ increases double logarithmically with the number of SUs as predicted by Theorem 3 . 


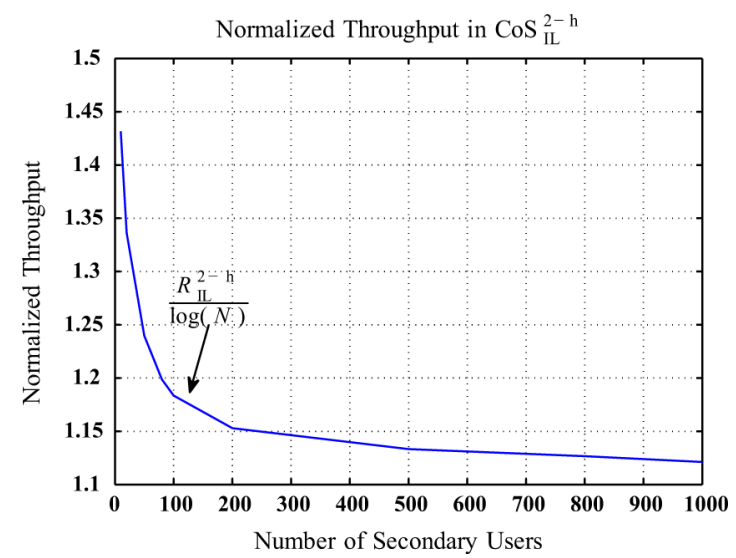

Fig. 7. Normalized throughput in $\operatorname{CoS}_{\mathrm{IL}}^{2-\mathrm{h}}$ with $Q_{\text {ave }}$ set to $0 \mathrm{~dB} . \alpha_{i}$ 's are uniformly distributed over $[0.2,1.8]$.

The next theorem establishes a similar extension of Theorem 2 to IL networks under the heterogeneity assumption.

Theorem 4: The sum-rate $R_{\mathrm{IL}}^{i-\mathrm{h}}(N)$ in $\mathrm{CoS}_{\mathrm{IL}}^{i-\mathrm{h}}$ scales according to $\lim _{N \rightarrow \infty} \frac{R_{\mathrm{IL}}^{i-\mathrm{h}}(N)}{\log (N)}=1$ for $i=1,2,4,5$. In $\operatorname{CoS}_{\mathrm{IL}}^{3-\mathrm{h}}$, the sum-rate is bounded as $\frac{1}{m_{\max }} \leq \liminf _{N \rightarrow \infty} \frac{R_{\mathrm{IL}}^{3-\mathrm{h}}(N)}{\log (N)} \leq$ $\limsup _{N \rightarrow \infty} \frac{R_{\mathrm{U}}^{3-\mathrm{h}}(N)}{\log (N)} \leq \frac{1}{m_{\min }}$.

Proof: Please refer to Appendix F.

In Appendix F, we give a proof of this theorem for $\mathrm{CoS}_{\mathrm{IL}}^{1-\mathrm{h}}$ and $\operatorname{CoS}_{\mathrm{IL}}^{3-\mathrm{h}}-\mathrm{COS}_{\mathrm{IL}}^{5-\mathrm{h}} \cdot \operatorname{CoS}_{\mathrm{IL}}^{2-\mathrm{h}}$ is a special case of $\operatorname{CoS}_{\mathrm{IL}}^{1-\mathrm{h}}$, and is obtained by setting Rician factors to zero. Theorem 4 shows that the secondary network throughput for IL networks still has a $\log (N)$-type behavior even in heterogeneous CoEs with different fading parameters for different SUs. In $\mathrm{CoS}_{\mathrm{IL}}^{3-\mathrm{h}}$, it is not possible to find the exact throughput scaling due to exponential effects of the Nakagami fading parameter on the effective number of users. Therefore, Theorem 4 only provides upper and lower bounds on the throughput scaling in this heterogeneous CoS.

In Fig. 7, we plot the normalized sum-rate of $\operatorname{CoS}_{\mathrm{IL}}^{2-\mathrm{h}}$ as a function of the number of SUs. In this figure, we set $Q_{\text {ave }}$ to $0 \mathrm{~dB}$, and we assume that $\alpha_{i}$ 's are uniformly distributed over $[0.2,1.8]$. The simulated sum-rate is obtained using the same averaging procedure as described above for the $\mathrm{CoS}_{\mathrm{PIL}}^{2-\mathrm{h}}$ case. As Fig. 7 shows, the sum-rate in $\operatorname{CoS}_{\mathrm{IL}}^{2-\mathrm{h}}$ scales according to $\log (N)$ with the number of SUs as predicted by Theorem 4 .

\section{CONCLUSION}

In this paper, we have focused on the secondary network sum-rate scaling in cognitive radio networks under different communication environments when SUs' transmission powers are optimally allocated. We have analyzed two different network types: PIL networks and IL networks. In a PIL network, SUs' transmissions are limited by both an average total power constraint and a constraint on the average interference that they cause to PUs. In an IL network, SUs' transmissions are only limited by an average interference constraint. In PIL networks, we have assumed Rayleigh fading model for the channels between SUs and the PBS, and Rayleigh, Rician or Nakagami fading models for the channels between SUs and the SBS. For PIL networks, our analysis shows that the secondary network throughput has a $\log \log (N)$-type behavior in all communication environments considered. For IL networks, in addition to these three communication environments, we have also considered Rayleigh fading model for the channels between SUs and the PBS, and Rician or Nakagami fading models for the channels between SUs and the SBS. For IL networks, our results show that the secondary network throughput scales according to $\log (N)$, which implies an exponential increase in the throughput scaling behavior when compared to PIL networks. These results have also been extended to heterogeneous communication environments in which different SUs experience different channel conditions. Finally, in some cases, our analysis has produced a new notion called effective number of users, which represents the effective number of SUs contributing to multiuser diversity gains.

\section{APPENDIX A \\ PROOF OF THEOREM 1}

Proof of Throughput Scaling in $\operatorname{CoS}_{P I L}^{1}$ : Recall $X_{i, N}=\frac{h_{i}}{\lambda_{N}+\mu_{N} g_{i}}$. When $N$ changes, the distribution of $X_{i, N}$ also changes. Therefore, the random variables of interest in this paper are in the form of triangular arrays, and the standard techniques from the extreme value theory cannot be applied directly [22]. To overcome this difficulty, we will first focus on a slightly modified sequence of random variables, and then argue why the same scaling results must hold for the original problem. To this end, let $Y_{i}(\lambda, \mu)=\frac{h_{i}}{\lambda+\mu g_{i}}$ for any given fixed $\lambda>0$ and $\mu>0$. Let $F(x)$ be the $\operatorname{CDF}$ of $Y_{i}(\lambda, \mu)$. Then, using the probability distribution function of $h_{i}$ as $\mathrm{e}^{-h_{i}}$ and that of $g_{i}$ as (Rician- $K) f\left(g_{i}\right)=(K+1) \mathrm{e}^{-K} \mathrm{e}^{-(K+1) g_{i}} I_{0}\left(2 \sqrt{K(K+1) g_{i}}\right)$, (where $I_{0}($.$) denotes the modified Bessel function of the first$ kind), one can easily show that $F(x)=P\left(Y_{i}(\lambda, \mu)<x\right)=$ $\int_{0}^{\infty}\left[1-\mathrm{e}^{-x(\lambda+\mu g)}\right] f(g) d g=1-\int_{0}^{\infty} \mathrm{e}^{-x(\lambda+\mu g)} f(g) d g$. Using the Rician- $K$ distribution for $g_{i}$, it then follows that $F(x)=1-(K+1) \mathrm{e}^{-K} \mathrm{e}^{-x \lambda} \int_{0}^{\infty} \mathrm{e}^{-\alpha g} I_{0}(2 \sqrt{\beta g}) d g$, where $\alpha=\mu x+K+1$ and $\beta=K(K+1)$. Using a formula for the integral in the above expression (see [35, p. 689]), one can then obtain $F(x)=1-\frac{K+1}{\mu x+K+1} \mathrm{e}^{-x\left(\lambda+\frac{\mu K}{\mu x+K+1}\right)}$. Let $Y_{N}^{\star}(\lambda, \mu)=\max _{1 \leq i \leq N} Y_{i}(\lambda, \mu) . F(x)$ satisfies the Von Mises conditions [22], i.e., $\lim _{x \rightarrow \infty} \frac{d}{d x}\left[\frac{1-F(x)}{f(x)}\right]=0$, where $f(x)$ denotes the probability density function (PDF) corresponding to $F(x)$. Therefore, we can find sequences of real numbers $\left\{a_{N}\right\}_{N=1}^{\infty}$ and $\left\{b_{N}\right\}_{N=1}^{\infty}$ such that $\frac{Y_{N}^{\star}(\lambda, \mu)-b_{N}}{a_{N}}$ converges in distribution to a Gumbel distributed random variable. That is, if $F_{N}^{\star}(x)$ is the CDF of $Y_{N}^{\star}(\lambda, \mu)$, then $\lim _{N \rightarrow \infty} F_{N}^{\star}\left(a_{N} x+b_{N}\right)=\exp \left(-\mathrm{e}^{-x}\right)$ for all $x \in \mathbb{R}$. Furthermore, normalizing constants $a_{N}$ and $b_{N}$ can be chosen to satisfy $F\left(b_{N}\right)=1-\frac{1}{N}$ and $a_{N}=\frac{1}{N f\left(b_{N}\right)}$, and they can be further expanded as $b_{N}=$ $\frac{1}{\lambda} \log \left(\frac{K+1}{\mathrm{e}^{K}} N\right)-\frac{1}{\lambda} \log \left(\frac{\mu}{\lambda} \log \left(\frac{K+1}{\mathrm{e}^{K}} N\right)\right)+O\left(\frac{\log \log (N)}{\log (N)}\right)$ and $a_{N}=\frac{1}{\lambda}\left(1-O\left(\frac{1}{\log (N)}\right)\right)$.

The following lemma, Lemma 3, characterizes the asymptotic behavior of $F_{N}^{\star}\left(a_{N} x+b_{N}\right)$. This lemma will be very helpful to estimate the tail probabilities lying under $F_{N}^{\star}(x)$. In Lemma 3 , we allow $x$ to vary with $N$ but do not show this relation explicitly. 
Lemma 3: For $x=O(\log (N))$, where $c_{N}(x)$ is given as (4) at the bottom of the page.

Proof: Using asymptotic expansions of $a_{N}$ and $b_{N}$, we have

$$
\begin{aligned}
& \frac{\mathrm{e}^{-\lambda\left(a_{N} x+b_{N}\right)}}{\mu\left(a_{N} x+b_{N}\right)+K+1} \\
& =\frac{\mathrm{e}^{K} \mathrm{e}^{-x}}{(K+1) N} \frac{\mathrm{e}^{O\left(\frac{x}{\log (N)}\right)-O\left(\frac{\log \log (N)}{\log (N)}\right)}}{1+O\left(\frac{x}{\log (N)}\right)-O\left(\frac{\log \log (N)}{\log (N)}\right)}
\end{aligned}
$$

and

$$
\begin{aligned}
& \frac{K(K+1)}{\mu\left(a_{N} x+b_{N}\right)+K+1} \\
& =\frac{K(K+1)}{\frac{\mu}{\lambda} x-O\left(\frac{x}{\log (N)}\right)+\frac{\mu}{\lambda} \log \left(\frac{K+1}{\mathrm{e}^{K}} N\right)-O(\log \log (N))} .
\end{aligned}
$$

Using the above expansions, we calculate the asymptotic expansion for $F\left(a_{N} x+b_{N}\right)$ as (6) at the bottom of the page.

The asymptotic expansion for $F_{N}^{\star}\left(a_{N} x+b_{N}\right)$ is obtained as $F_{N}^{\star}\left(a_{N} x+b_{N}\right)=\left(1-\frac{1}{N} c_{N}(x)\right)^{N}=\mathrm{e}^{-c_{N}(x)-O\left(\frac{c_{N}^{2}(x)}{N}\right)}$.

As an application of Lemma 3 , we can put $x=$ $\log \log \left(\frac{K+1}{\mathrm{e}^{K}} N\right)$ and $x=-\log \log \left(\frac{K+1}{\mathrm{e}^{K}} N\right)$ to obtain (7) at the bottom of the page. Equation (7) implies that $\frac{Y_{N}^{\star}(\lambda, \mu)}{\log (N)}$ converges in probability to $\frac{1}{\lambda}$ and $\frac{\log \left(Y_{N}^{\star}(\lambda, \mu)\right)}{\log \log (N)}$ converges in probability to 1 . Roughly speaking, these results intuitively suggest that the secondary network throughput should scale according to $\log \log (N)$ as $N$ tends to infinity. Since the convergence in probability does not always imply convergence in mean [33], we need some more work to establish the exact asymptotic behavior of $R_{\mathrm{PIL}}^{1}(N)$. To this end, let $\tilde{R}_{\mathrm{PIL}}^{1}(N, \lambda, \mu)=$
$\mathrm{E}\left[\log \left(Y_{N}^{\star}(\lambda, \mu)\right) 1_{\left\{Y_{N}^{\star}(\lambda, \mu) \geq 1\right\}}\right]$. It is not hard to prove that $\liminf _{N \rightarrow \infty} \frac{\tilde{R}_{\mathrm{PIL}}^{\mathrm{I}}(N, \lambda, \mu)}{\log \log (N)} \geq 1$. First, define the event $A=\left\{\frac{1}{\lambda} \log \left(\frac{K+1}{\mathrm{e}^{K}} N\right)-O(\log \log (N)) \leq Y_{N}^{\star}(\lambda, \mu) \leq\right.$ $\left.\frac{1}{\lambda} \log \left(\frac{K+1}{\mathrm{e}^{K}} N\right)-O(1)\right\}$. Then, $\operatorname{Pr}(A)=1-O\left(\frac{1}{\log (N)}\right)$. Therefore, for all $N$ large enough, $\tilde{R}_{\mathrm{PIL}}^{1}(N, \lambda, \mu)$ can be lower bounded as

$$
\begin{aligned}
\tilde{R}_{\mathrm{PIL}}^{1}(N, \lambda, \mu) \\
\geq \mathrm{E}\left[\log \left(Y_{N}^{\star}(\lambda, \mu)\right) 1_{A}\right] \\
=\left(\log \log \left(\frac{K+1}{\mathrm{e}^{K}} N\right)+\log \left(\frac{1}{\lambda}\right)+o(1)\right) \\
\quad \times\left(1-O\left(\frac{1}{\log (N)}\right)\right) \\
=\log \log \left(\frac{K+1}{\mathrm{e}^{K}} N\right)+\log \left(\frac{1}{\lambda}\right)+O(1) .
\end{aligned}
$$

The desired result immediately follows from (8).

To prove the other direction $\lim \sup _{N \rightarrow \infty} \frac{\tilde{R}_{\text {PIL }}^{1}(N, \lambda, \mu)}{\log \log (N)} \leq 1$, we will prove a stronger result given by the next lemma. In the next lemma, showing the effect of the Rician $K$ factor on $Y_{N}^{\star}(\lambda, \mu)$ explicitly will be helpful to provide effective number of users interpretation in Section IV.

Lemma 4: $\lim _{N \rightarrow \infty} \mathrm{E}\left[\frac{Y_{N}^{\star}(\lambda, \mu)}{\log \left(\frac{K+1}{\mathrm{e} K}\right)}\right]=\frac{1}{\lambda}$.
Proof: $\liminf _{N \rightarrow \infty} \mathrm{E}\left[\frac{Y_{N}^{\star}(\lambda, \mu)}{\log \left(\frac{K+1}{\mathrm{e} K}\right)}\right] \geq \frac{1}{\lambda}$ follows from Fatou's lemma [33] and the convergence of $\frac{Y_{N}^{\star}(\lambda, \mu)}{\log \left(\frac{K+1}{\mathrm{e}} N\right)}$ in probability to $\frac{1}{\lambda}$. To prove the other direction, we put $x=k \log \left(\frac{K+1}{\mathrm{e}^{K}} N\right)$ in Lemma 3 , where $k \geq 1$ is an integer.

$$
c_{N}(x)=\mathrm{e}^{-x}\left(\frac{\exp \left(O\left(\frac{x}{\log (N)}\right)-O\left(\frac{\log \log (N)}{\log (N)}\right)\right) \exp \left(\frac{K(K+1)}{\frac{\mu}{\lambda} x-O\left(\frac{x}{\log (N)}\right)+\frac{\mu}{\lambda} \log \left(\frac{K+1}{\mathrm{e} K} N\right)-O(\log \log (N))}\right)}{1+O\left(\frac{x}{\log (N)}\right)-O\left(\frac{\log \log (N)}{\log (N)}\right)}\right) .
$$

$$
\begin{aligned}
F\left(a_{N} x+b_{N}\right) & =1-\frac{1}{N} \mathrm{e}^{-x}\left(\frac{\exp \left(\left(O\left(\frac{x}{\log (N)}\right)-O\left(\frac{\log \log (N)}{\log (N)}\right)\right)\right) \exp \left(\frac{K(K+1)}{\frac{\mu}{\lambda} x-O\left(\frac{x}{\log (N)}\right)+\frac{\mu}{\lambda} \log \left(\frac{K+1}{\mathrm{e}^{K} N}\right)-O(\log \log (N))}\right)}{1+O\left(\frac{x}{\log (N)}\right)-O\left(\frac{\log \log (N)}{\log (N)}\right)}\right) \\
& =1-\frac{1}{N} c_{N}(x) .
\end{aligned}
$$

$$
\operatorname{Pr}\left\{\frac{1}{\lambda} \log \left(\frac{K+1}{\mathrm{e}^{K}} N\right)-O(\log \log (N)) \leq Y_{N}^{\star}(\lambda, \mu) \leq \frac{1}{\lambda} \log \left(\frac{K+1}{\mathrm{e}^{K}} N\right)-O(1)\right\}=1-O\left(\frac{1}{\log (N)}\right) .
$$


Then, $a_{N} x+b_{N}=\frac{k+1}{\lambda} \log \left(\frac{K+1}{\mathrm{e}^{K}} N\right)-O(\log \log (N))$ and $c_{N}(x)=\frac{\mathrm{e}^{O(k)}}{N^{k} O(k)} O(1)$. Thus, for $N$ large enough, we can write $\operatorname{Pr}\left\{Y_{N}^{\star}(\lambda, \mu) \leq \frac{k+1}{\lambda} \log \left(\frac{K+1}{\mathrm{e}^{K}} N\right)-O(\log \log (N))\right\}$

$$
=1-O\left(\frac{\mathrm{e}^{O(k)}}{N^{k} O(k)}\right) \text {. }
$$

Hence, $\operatorname{Pr}\left\{Y_{N}^{\star}(\lambda, \mu)>\frac{k+1}{\lambda} \log \left(\frac{K+1}{\mathrm{e}^{K}} N\right)\right\} \leq O\left(\frac{\mathrm{e}^{O(k)}}{N^{k} O(k)}\right)$. Take now a subsequence $N_{j}$ such that

$$
\limsup _{N \rightarrow \infty} \mathrm{E}\left[\frac{Y_{N}^{\star}(\lambda, \mu)}{\log \left(\frac{K+1}{\mathrm{e}^{K}} N\right)}\right]=\lim _{N_{j} \rightarrow \infty} \mathrm{E}\left[\frac{Y_{N_{j}}^{\star}(\lambda, \mu)}{\log \left(\frac{K+1}{\mathrm{e}^{K}} N_{j}\right)}\right]
$$

and $\lim _{N_{j} \rightarrow \infty} \frac{Y_{N_{j}}^{\star}(\lambda, \mu)}{\log \left(\frac{K+1}{K} N_{j}\right)}=\frac{1}{\lambda}$ almost surely. We can upper bound $\mathrm{E}\left[\frac{Y_{N_{j}}^{\star}(\lambda, \mu)}{\log \left(\frac{K+1}{\mathrm{e} K} N_{j}\right)}\right]$ as (10) at the bottom of the page. For $N_{j}$ large enough, the summation $\sum_{k=2}^{\infty} \frac{k+1}{\lambda} O\left(\frac{\mathrm{e}^{O(k)}}{N_{j}^{k-2} O(k)}\right)$ is always finite and a decreasing function of $N_{j}$. Thus, we have

$$
\begin{array}{r}
\mathrm{E}\left[\frac{Y_{N_{j}}^{\star}(\lambda, \mu)}{\log \left(\frac{K+1}{\mathrm{e}^{K}} N_{j}\right)}\right] \leq \mathrm{E}\left[\frac{Y_{N_{j}}^{\star}(\lambda, \mu)}{\log \left(\frac{K+1}{\mathrm{e}^{K}} N_{j}\right)} 1^{1}\left\{\frac{Y_{N_{j}}^{\star}(\lambda, \mu)}{\log \left(\frac{K+1}{\mathrm{e}^{K}} N_{j}\right)} \leq \frac{2}{\lambda}\right\}\right] \\
+O\left(\frac{1}{N_{j}}\right) .
\end{array}
$$

We finish the proof by taking the limits, and using the dominated convergence theorem.

By using Lemma 4, we can upper bound $\tilde{R}_{\mathrm{PIL}}^{1}(N, \lambda, \mu)$ for all $N$ large enough as

$$
\begin{aligned}
\tilde{R}_{\mathrm{PIL}}^{1}(N, \lambda, \mu) & \leq \mathrm{E}\left[\log \left(1+Y_{N}^{\star}(\lambda, \mu)\right)\right] \\
& \leq \log \left(1+\mathrm{E}\left[Y_{N}^{\star}(\lambda, \mu)\right]\right) \\
& \leq \log \left(1+\left(\frac{1}{\lambda}+\epsilon\right) \log \left(\frac{K+1}{\mathrm{e}^{K}} N\right)\right) \\
& =\log \log \left(\frac{K+1}{\mathrm{e}^{K}} N\right)+\log \left(\frac{1}{\lambda}+\epsilon\right)+O(1),
\end{aligned}
$$

where $\epsilon>0$, and (a) follows from Jensen's inequality. Therefore, we have $\lim \sup _{N \rightarrow \infty} \frac{\tilde{R}_{\text {PIII }}(N, \lambda, \mu)}{\log \log (N)} \leq 1$, which implies $\lim _{N \rightarrow \infty} \frac{\tilde{R}_{\text {PII }}^{1}(N, \lambda, \mu)}{\log \log (N)}=1$ for all $\lambda>0$ and $\mu>0$.
Now, consider the original setup, and let $I_{N}=$ $\arg \max _{1 \leq i \leq N} X_{i, N}$. Assume that $\liminf _{N \rightarrow \infty} \mu_{N}>0$. Otherwise, all arguments (above and below) can be repeated by setting $\mu=0$ when necessary. For PIL networks, both constraints are active, i.e., $\lambda_{N}>0$ and $\mu_{N}>0$, and therefore we have $P_{\text {ave }}=\mathrm{E}\left[\left(\frac{1}{\lambda_{N}+\mu_{N} g_{I_{N}}}-\frac{1}{h_{I_{N}}}\right)^{+}\right]$and $Q_{\text {ave }}=\mathrm{E}\left[g_{I_{N}}\left(\frac{1}{\lambda_{N}+\mu_{N} g_{I_{N}}}-\frac{1}{h_{I_{N}}}\right)^{+}\right]$. These equalities imply that $\lambda_{N} \leq \frac{1}{P_{\text {ave }}}$ and $\mu_{N} \leq \frac{1}{Q_{\text {ave }}}$ for all $N \geq 1$. We also argue that $\lim \inf _{N \rightarrow \infty} \lambda_{N}>0$. Otherwise, for any $\epsilon>0$, the transmission power $P_{I_{N}}^{\star}$ becomes larger than $\frac{\left(Y_{N}^{\star}\left(\epsilon, \frac{1}{Q_{\text {ave }}}\right)-1\right)^{+}}{h_{N}^{+}}$ for all $N$ large enough, where $h_{N}^{\star}=\max _{1 \leq i \leq N} h_{i}$. As shown above, $Y_{N}^{\star}\left(\epsilon, \frac{1}{Q_{\text {ave }}}\right)$ scales according to $\frac{1}{\epsilon} \log (N)$. $h_{N}^{\star}$ scales according to $\log (N)$. Thus, the transmission power becomes larger than $\frac{1}{\epsilon}$ with probability arbitrarily close to one for all $N$ large enough, which violates the average total transmission power constraint for $\epsilon$ small enough. Therefore, we can find $\epsilon>0$ and $\delta>0$ such that

$$
\tilde{R}_{\mathrm{PIL}}^{1}\left(N, \frac{1}{P_{\mathrm{ave}}}, \frac{1}{Q_{\mathrm{ave}}}\right) \leq R_{\mathrm{PIL}}^{1}(N) \leq \tilde{R}_{\mathrm{PIL}}^{1}(N, \epsilon, \delta)
$$

for all $N$ large enough. The proof is complete since $\lim _{N \rightarrow \infty} \frac{\tilde{R}_{\mathrm{PI}}^{1}(N, \lambda, \mu)}{\log \log (N)}=1$ for all $\lambda>0$ and $\mu>0$.

Proof of Throughput Scaling in $\operatorname{CoS}_{P I L}^{3}$ : We will only provide key proof ideas since the proof of throughput scaling in $\operatorname{CoS}_{\mathrm{PIL}}^{3}$ is similar to the one given for $\operatorname{CoS}_{\mathrm{PIL}}^{1}$. For any $\lambda>0$ and $\mu>0$, let $Y_{i}(\lambda, \mu)=\frac{h_{i}}{\lambda+\mu g_{i}}$ and $Y_{N}^{\star}(\lambda, \mu)=\max _{1<i<N} Y_{i}(\lambda, \mu)$ as above. After some analysis, the $\operatorname{CDF} F(x)$ of $Y_{i}(\lambda, \mu)$ in this case can be derived as $F(x)=1-\mathrm{e}^{-\lambda x}\left(\frac{m}{m+\mu x}\right)^{m}$, where $m \geq 0.5$ is the Nakagami fading parameter. $F(x)$ satisfies the Von Mises conditions, i.e., $\lim _{x \rightarrow \infty} \frac{d}{d x} \frac{1-F(x)}{f(x)}=0$, where $f(x)$ is the PDF corresponding to $F(x)$. Therefore, there exist sequences of real numbers $\left\{a_{N}\right\}_{N=1}^{\infty}$ and $\left\{b_{N}\right\}_{N=1}^{\infty}$ such that $\frac{Y_{N}^{\star}(\lambda, \mu)-b_{N}}{a_{N}}$ converges in distribution to a Gumbel distributed random variable. $a_{N}$ and $b_{N}$ can be chosen to satisfy $F\left(b_{N}\right)=1-\frac{1}{N}$ and $a_{N}=\frac{1}{N f\left(b_{N}\right)}$, which can be further expanded as $b_{N}=\frac{1}{\lambda} \log (N)-\frac{m}{\lambda} \log \left(\frac{\mu}{\lambda m} \log (N)\right)+O\left(\frac{\log \log (N)}{\log (N)}\right)$ and $a_{N}=\frac{1}{\lambda}-O\left(\frac{1}{\log (N)}\right)$. Let $F_{N}^{\star}(x)$ be the CDF of $Y_{N}^{\star}(\lambda, \mu)$. The following lemma characterizes the asymptotic behavior of $F_{N}^{\star}\left(a_{N} x+b_{N}\right)$. In Lemma 5, we will allow $x$ to vary with $N$ but do not show this relation explicitly.

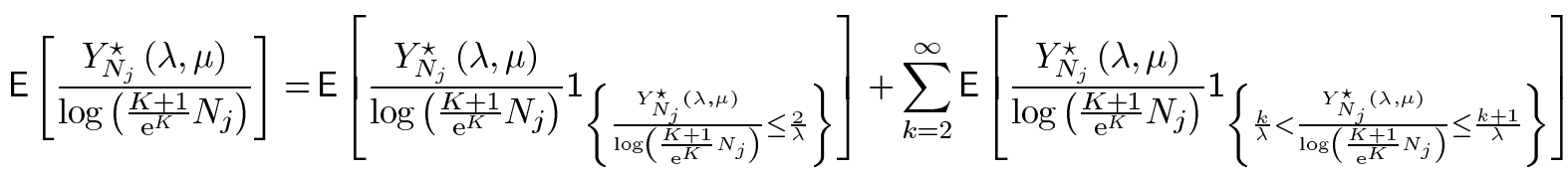

$$
\begin{aligned}
& \leq \mathrm{E}\left[\frac{Y_{N_{j}}^{\star}(\lambda, \mu)}{\log \left(\frac{K+1}{\mathrm{e}^{K}} N_{j}\right)}\left\{\frac{Y_{N_{j}}^{\star}(\lambda, \mu)}{\log \left(\frac{K+1}{\left.\mathrm{e}^{K} N_{j}\right)}\right.} \leq \frac{2}{\lambda}\right\}\right]+\frac{1}{N_{j}} \sum_{k=2}^{\infty} \frac{k+1}{\lambda} O\left(\frac{\mathrm{e}^{O(k)}}{N_{j}^{k-2} O(k)}\right) .
\end{aligned}
$$


Lemma 5: For $x=O(\log (N)), F_{N}^{\star}\left(a_{N} x+b_{N}\right)=$ $\mathrm{e}^{-c_{N}(x)-O\left(\frac{c_{N}^{2}(x)}{N}\right)}$, where

$$
c_{N}(x)=\mathrm{e}^{-x} \frac{\mathrm{e}^{-O\left(\frac{\log \log (N)}{\log (N)}\right)} \mathrm{e}^{O\left(\frac{x}{\log (N)}\right)}}{\left[1+O\left(\frac{x}{\log (N)}\right)-O\left(\frac{\log \log (N)}{\log (N)}\right)\right]^{m}} .
$$

Proof: Using asymptotic expansions of $a_{N}$ and $b_{N}$, we have

$\mathrm{e}^{-\lambda\left(a_{N} x+b_{N}\right)}$

$$
=\mathrm{e}^{-x} \mathrm{e}^{O\left(\frac{x}{\log (N)}\right)} \frac{1}{N}\left(\frac{\mu}{\lambda m} \log (N)\right)^{m} \mathrm{e}^{-O\left(\frac{\log \log (N)}{\log (N)}\right)}
$$

and

$$
\begin{aligned}
\mu\left(a_{N} x+b_{N}\right)+m= & m+\frac{\mu}{\lambda} x-O\left(\frac{x}{\log (N)}\right) \\
& +\frac{\mu}{\lambda} \log (N)-\frac{\mu m}{\lambda} \log \left(\frac{\mu}{\lambda m} \log (N)\right) \\
& +O\left(\frac{\log \log (N)}{\log (N)}\right) .
\end{aligned}
$$

Using expansions for $\mathrm{e}^{-\lambda\left(a_{N} x+b_{N}\right)}$ and $\mu\left(a_{N} x+b_{N}\right)+m$ given above, $\mathrm{e}^{-\lambda\left(a_{N} x+b_{N}\right)}\left(\frac{m}{\mu\left(a_{N} x+b_{N}\right)+m}\right)^{m}$ can be written as

$$
\begin{aligned}
\mathrm{e}^{-\lambda\left(a_{N} x+b_{N}\right)}\left(\frac{m}{\mu\left(a_{N} x+b_{N}\right)+m}\right)^{m} \\
=\frac{1}{N} \mathrm{e}^{-x} \frac{\mathrm{e}^{-O\left(\frac{\log \log (N)}{\log (N)}\right)} \mathrm{e}^{O\left(\frac{x}{\log (N)}\right)}}{\left(1+O\left(\frac{x}{\log (N)}\right)-O\left(\frac{\log \log (N)}{\log (N)}\right)\right)^{m}} \\
=\frac{1}{N} c_{N}(x) .
\end{aligned}
$$

The asymptotic expansion for $F_{N}^{\star}\left(a_{N} x+b_{N}\right)$ is obtained as $F_{N}^{\star}\left(a_{N} x+b_{N}\right)=\left(1-\frac{1}{N} c_{N}(x)\right)^{N}=\mathrm{e}^{-c_{N}(x)-O\left(\frac{c_{N}^{2}(x)}{N}\right)}$, which completes the proof.

The rest of the proof follows the similar arguments that we used in the throughput scaling proof for $R_{\mathrm{PIL}}^{1}(N)$. Therefore, we skip them to avoid repetitions.

\section{APPENDIX B}

\section{CONVERGENCE BEHAVIOR OF $\lambda_{N}$}

In this appendix, we will establish an important convergence behavior for the Lagrange multipliers $\lambda_{N}$ for all $\mathrm{CoS}_{\mathrm{PIL}}^{i}$, $i \in\{1,2,3\}$. As we claim in Section IV, this property will imply that $P_{\text {ave }}$ has logarithmic effects on the secondary network throughput. $\frac{1}{P_{\text {ave }}}$.

Lemma 6: For all $\mathrm{CoS}_{\mathrm{PIL}}^{i}, i \in\{1,2,3\}, \lim _{N \rightarrow \infty} \lambda_{N}=$

Proof: We define $h_{I_{N}}, g_{I_{N}}, h_{N}^{\star}$ and $Y_{N}^{\star}(\lambda, \mu)$ as in Appendix A. From Appendix A, we have $\lambda_{N} \leq \frac{1}{P_{\text {ave }}}$. Thus, $\lim \sup _{N \rightarrow \infty} \lambda_{N} \leq \frac{1}{P_{\text {ave }}}$. Now, we show that $\liminf _{N \rightarrow \infty} \lambda_{N} \geq \frac{1}{P_{\text {ave }}}$ as follows:

$$
\begin{aligned}
P_{\text {ave }} & =\mathrm{E}\left[\left(\frac{1}{\lambda_{N}+\mu_{N} g_{I_{N}}}-\frac{1}{h_{I_{N}}}\right)^{+}\right] \\
& =\frac{1}{\lambda_{N}} \mathrm{E}\left[\left(\frac{1}{1+\frac{\mu_{N}}{\lambda_{N}} g_{I_{N}}}-\frac{\lambda_{N}}{h_{I_{N}}}\right)^{+}\right]
\end{aligned}
$$

$$
\begin{aligned}
& =\frac{1}{\lambda_{N}} \mathrm{E}\left[\left(\frac{\frac{h_{I_{N}}}{1+\frac{\mu_{N}}{\lambda_{N}} g_{I_{N}}}-\lambda_{N}}{h_{I_{N}}}\right)^{+}\right] \\
& \geq \frac{1}{\lambda_{N}} \mathrm{E}\left[\left(\frac{\frac{h_{I_{N}}}{1+\frac{\mu_{N}}{\lambda_{N}} g_{I_{N}}}-\lambda_{N}}{h_{N}^{\star}}\right)^{+}\right] \\
& =\frac{1}{\lambda_{N}} \mathrm{E}\left[\left(\frac{Y_{N}^{\star}\left(1, \frac{\mu_{N}}{\lambda_{N}}\right)}{h_{N}^{\star}}-\frac{\lambda_{N}}{h_{N}^{\star}}\right)^{+}\right] .
\end{aligned}
$$

Pick $\epsilon>0$ such that $\liminf _{N \rightarrow \infty} \lambda_{N} \geq \epsilon$. For $N$ large enough, we have $0 \leq \frac{\mu_{N}}{\lambda_{N}} \leq \frac{1}{\epsilon Q_{\text {ave }}}$ and $Y_{N}^{\star}\left(1, \frac{1}{\epsilon Q_{\text {ave }}}\right) \leq Y_{N}^{\star}\left(1, \frac{\mu_{N}}{\lambda_{N}}\right) \leq$ $Y_{N}^{\star}(1,0)$. When $\mu_{N}=0$ (i.e., in the absence of any average interference constraint), $\frac{Y_{N}^{\star}(1,0)}{\log (N)} \stackrel{i . p .}{\longrightarrow} 1$ since $Y_{N}^{\star}(1,0)=h_{N}^{\star}$ and $h_{N}^{\star}$ scales according to $\log (N)$ in all CoSs considered. From Appendix A, we also have $\frac{Y_{N}^{\star}\left(1, \frac{1}{\epsilon Q_{\text {ave }}}\right)}{\log (N)} \stackrel{i . p .}{\longrightarrow} 1$. Therefore, $\quad \frac{Y_{N}^{\star}\left(1, \frac{\mu_{N}}{\lambda_{N}}\right)}{\log (N)} \stackrel{i . p .}{\longrightarrow} 1$ and $\frac{Y_{N}^{\star}\left(1, \frac{\mu_{N}}{\lambda_{N}}\right)}{h_{N}^{\star}} \stackrel{i . p .}{\longrightarrow} 1$. We also have $\frac{\lambda_{N}}{h_{N}^{\star}} \stackrel{i . p .}{\longrightarrow} 0$. Thus, $\left(\frac{Y_{N}^{\star}\left(1, \frac{\mu_{N}}{\lambda_{N}}\right)}{h_{N}^{\star}}-\frac{\lambda_{N}}{h_{N}^{\star}}\right) \stackrel{i . p .}{\longrightarrow} 1$ and $\left(\frac{Y_{N}^{\star}\left(1, \frac{\mu_{N}}{\lambda_{N}}\right)}{h_{N}^{\star}}-\frac{\lambda_{N}}{h_{N}^{\star}}\right)^{+} \stackrel{i . p .}{\longrightarrow} 1$. Using (15), $\lambda_{N}$ can be lower bounded as

$$
\lambda_{N} \geq \frac{1}{P_{\text {ave }}} \mathrm{E}\left[\left(\frac{Y_{N}^{\star}\left(1, \frac{\mu_{N}}{\lambda_{N}}\right)}{h_{N}^{\star}}-\frac{\lambda_{N}}{h_{N}^{\star}}\right)^{+}\right] .
$$

Taking liminf as $N \rightarrow \infty$ on both sides and applying Fatou's lemma, we have

$$
\begin{aligned}
\liminf _{N \rightarrow \infty} \lambda_{N} & \geq \frac{1}{P_{\text {ave }}} \liminf _{N \rightarrow \infty} \mathrm{E}\left[\left(\frac{Y_{N}^{\star}\left(1, \frac{\mu_{N}}{\lambda_{N}}\right)}{h_{N}^{\star}}-\frac{\lambda_{N}}{h_{N}^{\star}}\right)^{+}\right] \\
& \geq \frac{1}{P_{\text {ave }}}
\end{aligned}
$$

which completes the proof.

\section{APPENDIX C \\ PROOF OF THEOREM 2}

Proof of Throughput Scaling in $\mathrm{CoS}_{\mathrm{IL}}^{1}$ : Recall $X_{i, N}=\frac{h_{i}}{\mu_{N} g_{i}}$ and $X_{N}^{\star}=\max _{1 \leq i \leq N} X_{i, N}$. Similar to our proofs above, we introduce the auxiliary random variables $Y_{i}(\mu)=\frac{h_{i}}{\mu g_{i}}$ and $Y_{N}^{\star}(\mu)=\max _{1 \leq i \leq N} Y_{i}(\mu)$ for a fixed $\mu>0$ to prove the throughput scaling result in $\operatorname{CoS}_{\mathrm{IL}}^{1}$. Let $F(x)$ be the CDF of $Y_{i}(\mu)$, and $F_{N}^{\star}(x)$ be the CDF of $Y_{N}^{\star}(\mu)$. Note that, using a similar derivation as in the Proof of Theorem 1 in Appendix A, or by simply putting $\lambda=0$ in the expression for $P\left(\frac{h_{i}}{\lambda+\mu g_{i}}<x\right)$ in Appendix A, $F(x)$ can be derived as $F(x)=1-\frac{(K+1) \mathrm{e}^{-K}}{\mu x+K+1} \mathrm{e}^{\frac{K(K+1)}{\mu x+K+1}} \cdot F(x)$ satisfies $\lim _{x \rightarrow \infty} \frac{x f(x)}{1-F(x)}=1$, where $f(x)$ denotes the PDF corresponding to $F(x)$. Therefore, we can find a sequence of real numbers $\left\{a_{N}\right\}_{N=1}^{\infty}$ such that $\frac{Y_{N}^{\star}(\mu)}{a_{N}}$ converges in distribution to a Frechet distributed random variable. That is, for all $x>0$, we have $\lim _{N \rightarrow \infty} F_{N}^{\star}\left(a_{N} x\right)=\exp \left(-\frac{1}{x}\right)$. The normalizing 
constants $a_{N}$ can be chosen to satisfy $F\left(a_{N}\right)=1-\frac{1}{N}$, which implies the asymptotic expansion

$$
a_{N}=\frac{K+1}{\mu \mathrm{e}^{K}} \mathrm{e}^{\frac{K}{N} \mathrm{e}^{K}} N-\frac{K+1}{\mu}-O\left(\frac{1}{N}\right) .
$$

The following lemma characterizes the asymptotic behavior of $F_{N}^{\star}\left(a_{N} x\right)$. In this lemma, we allow $x$ to vary with $N$ but do not show this relation explicitly.

Lemma 7: For $x=O(\log (N)), F_{N}^{\star}\left(a_{N} x\right)=$ $\mathrm{e}^{-c_{N}(x)-O\left(\frac{c_{N}^{2}(x)}{N}\right)}$, where $c_{N}(x)$ is given by

$$
c_{N}(x)=\frac{\exp \left(\frac{K}{\exp \left(-K+\frac{K}{N} \mathrm{e}^{K}\right) N x-O(x)+O(1)}\right)}{\exp \left(\frac{K}{N} \mathrm{e}^{K}\right) x-O\left(\frac{x}{N}\right)+O\left(\frac{1}{N}\right)} .
$$

Proof: Using the asymptotic expansion of $a_{N}$, we have

$$
\begin{aligned}
\exp & \left(\frac{K(K+1)}{\mu a_{N} x+K+1}\right) \\
\quad= & \exp \left(\frac{K}{\exp \left(-K+\frac{K}{N} \mathrm{e}^{K}\right) N x-O(x)+O(1)}\right)
\end{aligned}
$$

and

$$
\begin{aligned}
& \frac{1}{\mu a_{N} x+K+1} \\
& \quad=\frac{1}{(K+1) \mathrm{e}^{-K} N} \frac{1}{\exp \left(\frac{K}{N} \mathrm{e}^{K}\right) x-O\left(\frac{x}{N}\right)+O\left(\frac{1}{N}\right)} .
\end{aligned}
$$

Therefore, we have

$$
\begin{aligned}
& \frac{(K+1) \mathrm{e}^{-K}}{\mu a_{N} x+K+1} \exp \left(\frac{K(K+1)}{\mu a_{N} x+K+1}\right) \\
& =\frac{1}{N} \frac{\exp \left(\frac{K}{\exp \left(-K+\frac{K}{N} \mathrm{e}^{K}\right) N x-O(x)+O(1)}\right)}{\exp \left(\frac{K}{N} \mathrm{e}^{K}\right) x-O\left(\frac{x}{N}\right)+O\left(\frac{1}{N}\right)} \\
& =\frac{1}{N} c_{N}(x) .
\end{aligned}
$$

Finally, the asymptotic expansion of $F_{N}^{\star}\left(a_{N} x\right)$ is obtained as $F_{N}^{\star}\left(a_{N} x\right)=\left(1-\frac{1}{N} c_{N}(x)\right)^{N}=\mathrm{e}^{-c_{N}(x)-O\left(\frac{c_{N}^{2}(x)}{N}\right)}$.
As an application of Lemma 7, we put $x=\frac{\log (N)}{\exp \left(\frac{K}{N} \mathrm{e}^{K}\right)}$ and $x=\frac{1}{\exp \left(\frac{K}{N} \mathrm{e}^{K}\right) \log (N)}$ to obtain (18) at the bottom of the page, which implies that $\frac{\log \left(Y_{N}^{\star}(\mu)\right)}{\log (N)}$ converges in probability to 1 . Similar to our proof for PIL networks, (18) intuitively suggests that the secondary network throughput should scale according to $\log (N)$ for IL networks. Next, we will make this intuition rigorous.

To this end, let $\tilde{R}_{\mathrm{IL}}^{1}(N, \mu)=\mathrm{E}\left[\log \left(Y_{N}^{\star}(\mu)\right) 1_{\left\{Y_{N}^{\star}(\mu) \geq 1\right\}}\right]$. It is easy to prove $\liminf _{N \rightarrow \infty} \frac{\tilde{R}_{\mathrm{II}}^{1}(N, \mu)}{\log (N)} \geq 1$. We first define the event [see (19) at the bottom of the page]. Then, $\operatorname{Pr}(A)=$ $1-O\left(\frac{1}{\log (N)}\right)$. Therefore, for all $N$ large enough, $\tilde{R}_{\mathrm{IL}}^{1}(N, \mu)$ can be lower bounded as

$$
\begin{aligned}
& \tilde{R}_{\mathrm{IL}}^{1}(N, \mu) \geq \mathrm{E}\left[\log \left(Y_{N}^{\star}(\mu)\right) 1_{A}\right] \\
& \geq \log \left(\frac{K+1}{\mu \mathrm{e}^{K}} N\right)\left(1-O\left(\frac{\log \log (N)}{\log (N)}\right)\right) .
\end{aligned}
$$

The desired result immediately follows from (20). To prove the other direction, i.e., $\lim \sup _{N \rightarrow \infty} \frac{\tilde{R}_{\mathrm{IL}}^{1}(N, \mu)}{\log (N)} \leq 1$, we define the events

$$
\begin{aligned}
B & =\left\{1 \leq Y_{N}^{\star}(\mu) \leq \frac{K+1}{\mu \mathrm{e}^{K}} N \log ^{2}(N)\right\} \\
B_{k} & =\left\{\frac{K+1}{\mu \mathrm{e}^{K}} N \log ^{k}(N)<Y_{N}^{\star}(\mu) \leq \frac{K+1}{\mu \mathrm{e}^{K}} N \log ^{k+1}(N)\right\}
\end{aligned}
$$

for $k \geq 2$. By using Lemma 7, we can estimate probabilities $\operatorname{Pr}\left(B_{k}\right)$ as $\operatorname{Pr}\left(B_{k}\right) \leq O\left(\frac{1}{\log ^{k}(N)}\right)$. Then, $\tilde{R}_{\mathrm{IL}}^{1}(N, \mu)$ is upper bounded as

$$
\begin{aligned}
& \tilde{R}_{\mathrm{IL}}^{1}(N, \mu) \\
& =\mathrm{E}\left[\log \left(Y_{N}^{\star}(\mu)\right) 1_{B}\right]+\sum_{k=2}^{\infty} \mathrm{E}\left[\log \left(Y_{N}^{\star}(\mu)\right) 1_{B_{k}}\right] \\
& \leq \log \left(\frac{K+1}{\mu \mathrm{e}^{K}} N \log ^{2}(N)\right) \\
& \quad+\frac{\log \log (N)}{\log (N)} \sum_{k=2}^{\infty} O\left(\frac{O(k)}{\log ^{k-1}(N)}\right)+\sum_{k=2}^{\infty} O\left(\frac{1}{\log ^{k-1}(N)}\right) .
\end{aligned}
$$

$$
\begin{aligned}
& \operatorname{Pr}\left\{\frac{1}{\mu \log (N)}\left(\frac{(K+1) N}{\mathrm{e}^{K}}-\frac{K+1}{\exp \left(\frac{K}{N} \mathrm{e}^{K}\right)}-O\left(\frac{1}{N}\right)\right)<Y_{N}^{\star}(\mu)\right. \\
& \left.\leq \frac{\log (N)}{\mu}\left(\frac{(K+1) N}{\mathrm{e}^{K}}-\frac{K+1}{\exp \left(\frac{K}{N} \mathrm{e}^{K}\right)}-O\left(\frac{1}{N}\right)\right)\right\}=1-O\left(\frac{1}{\log (N)}\right)
\end{aligned}
$$

$$
\begin{aligned}
& A=\left\{\frac{1}{\mu \log (N)}\left(\frac{(K+1) N}{\mathrm{e}^{K}}-\frac{K+1}{\exp \left(\frac{K}{N} \mathrm{e}^{K}\right)}-O\left(\frac{1}{N}\right)\right)<Y_{N}^{\star}(\mu)\right. \\
& \left.\leq \frac{\log (N)}{\mu}\left(\frac{(K+1) N}{\mathrm{e}^{K}}-\frac{K+1}{\exp \left(\frac{K}{N} \mathrm{e}^{K}\right)}-O\left(\frac{1}{N}\right)\right)\right\} .
\end{aligned}
$$


For $\quad N \quad$ large enough, $\quad \sum_{k=2}^{\infty} O\left(\frac{O(k)}{\log ^{k-1}(N)}\right) \quad$ and $\sum_{k=2}^{\infty} O\left(\frac{1}{\log ^{k-1}(N)}\right)$ are always finite and decreasing functions of $N$. Thus, we have

$$
\tilde{R}_{\mathrm{IL}}^{1}(N, \mu) \leq \log \left(\frac{K+1}{\mu \mathrm{e}^{K}} N\right)\left(1+O\left(\frac{\log \log (N)}{\log (N)}\right)\right) .
$$

Therefore, we have $\lim \sup _{N \rightarrow \infty} \frac{\tilde{R}_{\mathrm{IL}}^{1}(N, \mu)}{\log (N)} \leq 1$, which implies that $\lim _{N \rightarrow \infty} \frac{\tilde{R}_{\mathrm{IL}}^{1}(N, \mu)}{\log (N)}=1$ for all $\mu>0$.

Now, consider the original setup. For IL networks, the interference constraint is active, i.e., $\mu_{N}>0$, and therefore $\mu_{N} Q_{\text {ave }}=\mathrm{E}\left[\left(1-\frac{1}{X_{N}^{\star}}\right)^{+}\right]$holds. Since $\left(1-\frac{1}{X_{N}^{\star}}\right)^{+} \leq 1$, this identity implies $\mu_{N} \leq \frac{1}{Q_{a v}}$ for all $N \geq 1$. We also argue that $\liminf _{N \rightarrow \infty} \mu_{N}>0$. Otherwise, we can find a subsequence $\left\{N_{j}\right\}_{j=1}^{\infty}$ such that $\frac{1}{Y_{N_{j}}^{\star}(1)}$ converges to 0 almost surely, $\lim _{N_{j} \rightarrow \infty} \mu_{N_{j}}=0$. Then, the average interference caused to the PBS becomes larger than $\frac{1}{\mu_{N_{j}}} \mathrm{E}\left[\left(1-\frac{1}{Y_{N_{j}}^{\star}(1)}\right)^{+}\right]$for all $N_{j}$ large enough. By using dominated convergence theorem, we have $\lim _{N_{j} \rightarrow \infty} \mathrm{E}\left[\left(1-\frac{1}{Y_{N_{j}}^{\star}(1)}\right)^{+}\right]=1$, which implies $\lim _{N_{j} \rightarrow \infty} \frac{1}{\mu_{N_{j}}} \mathrm{E}\left[\left(1-\frac{1}{Y_{N_{j}}^{\star}(1)}\right)^{+}\right]=\infty$, and the average interference constraint is violated. Therefore, we can find $\epsilon>0$ small enough such that

$$
\tilde{R}_{\mathrm{IL}}^{1}\left(N, \frac{1}{Q_{\text {ave }}}\right) \leq R_{\mathrm{IL}}^{1}(N) \leq \tilde{R}_{\mathrm{IL}}^{1}(N, \epsilon)
$$

for all $N$ large enough. The proof is complete since $\lim _{N \rightarrow \infty} \frac{\tilde{R}_{\mathrm{IL}}^{1}(N, \mu)}{\log (N)}=1$ for all $\mu>0$.

Proof of Throughput Scaling in $\operatorname{CoS}_{\mathrm{IL}}^{3}$ : For any $\mu>0$, let $Y_{i}(\mu)=\frac{h_{i}}{\mu g_{i}}$ and $Y_{N}^{\star}(\mu)=\max _{1 \leq i \leq N} Y_{i}(\mu)$ as above. After some analysis, the $\mathrm{CDF} F(x)$ of $Y_{i}(\mu)=\frac{h_{i}}{\mu g_{i}}$ in this case can be derived as $F(x)=1-\left(\frac{m}{\mu x+m}\right)^{m}$, where $m \geq 0.5$ is the Nakagami fading parameter. Let $F_{N}^{\star}(x)$ be the CDF of $Y_{N}^{\star}(\mu)$. $F(x)$ satisfies $\lim _{x \rightarrow \infty} \frac{x f(x)}{1-F(x)}=1$, where $f(x)$ is the PDF corresponding to the $F(x)$. Therefore, we can find a sequence of real numbers $\left\{a_{N}\right\}_{N=1}^{\infty}$ such that $\frac{Y_{N}^{\star}(\mu)}{a_{N}}$ converges in distribution to a Frechet distributed random variable. The normalizing constants $a_{N}$ can be chosen to satisfy $F\left(a_{N}\right)=1-\frac{1}{N}$, which implies $a_{N}=\frac{m N^{\frac{1}{m}}}{\mu}-O(1)$. The following lemma characterizes the asymptotic behavior of $F_{N}^{\star}\left(a_{N} x\right)$.

Lemma 8: For $x=O(\log (N)), F_{N}^{\star}\left(a_{N} x\right)=$ $\mathrm{e}^{-c_{N}(x)-O\left(\frac{c_{N}^{2}(x)}{N}\right)}$, where $c_{N}(x)$ is given by

$$
c_{N}(x)=\frac{1}{x^{m}\left(1-O\left(\frac{1}{N^{\frac{1}{m}}}\right)+\frac{1}{N^{\frac{1}{m}} x}\right)^{m}} .
$$

Proof: Using the asymptotic expansion of $a_{N}$, we have $\left(\frac{m}{\mu a_{N} x+m}\right)^{m}=\frac{1}{N x^{m}\left(1-O\left(\frac{1}{N^{\frac{1}{m}}}\right)+\frac{1}{N^{\frac{1}{m}} x}\right)^{m}}$. We use this result to find an asymptotic expansion for $F\left(a_{N} x\right)$ as

$$
\begin{aligned}
F\left(a_{N} x\right) & =1-\left(\frac{m}{\mu a_{N} x+m}\right)^{m} \\
& =1-\frac{1}{N x^{m}\left(1-O\left(\frac{1}{N^{\frac{1}{m}}}\right)+\frac{1}{N^{\frac{1}{m}} x}\right)^{m}}=1-\frac{1}{N} c_{N}(x) .
\end{aligned}
$$

Finally, the asymptotic expansion of $F_{N}^{\star}\left(a_{N} x\right)$ is obtained as $F_{N}^{\star}\left(a_{N} x\right)=\left(1-\frac{1}{N} c_{N}(x)\right)^{N}=\mathrm{e}^{-c_{N}(x)-O\left(\frac{c_{N}^{2}(x)}{N}\right)}$.

Using Lemma 8 and following the same steps as in the throughput scaling proof given for $\operatorname{CoS}_{\mathrm{IL}}^{1}$, we obtain

$$
\begin{aligned}
\log \left(\frac{m}{\mu} N^{\frac{1}{m}}\right) & \left(1-O\left(\frac{\log \log (N)}{\log (N)}\right)\right) \leq \tilde{R}_{\mathrm{IL}}^{3}(N, \mu) \\
\leq & \log \left(\frac{m}{\mu} N^{\frac{1}{m}}\right)\left(1+O\left(\frac{\log \log (N)}{\log (N)}\right)\right)
\end{aligned}
$$

for any $\mu>0$. It also holds that $\liminf _{N \rightarrow \infty} \mu_{N}>0$. Since

$$
\tilde{R}_{\mathrm{IL}}^{3}\left(N, \frac{1}{Q_{\text {ave }}}\right) \leq R_{\mathrm{IL}}^{3}(N) \leq \tilde{R}_{\mathrm{IL}}^{3}(N, \epsilon)
$$

for $\epsilon>0$ small enough, we have $\lim _{N \rightarrow \infty} \frac{R_{\mathrm{LI}}^{3}(N)}{\log (N)}=\frac{1}{m}$.

Proof of Throughput Scaling in $\operatorname{CoS}_{\mathrm{IL}}^{4}$ : For any $\mu>0$, let $Y_{i}(\mu)=\frac{h_{i}}{\mu g_{i}}$ and $Y_{N}^{\star}(\mu)=\max _{1 \leq i \leq N} Y_{i}(\mu)$ as above. The $\mathrm{CDF}$ of $Y_{i}(\mu)$ is given by $F(x)=\left(\frac{m \mu x}{m \mu x+1}\right)^{m}$ in this case. $F(x)$ satisfies $\lim _{x \rightarrow \infty} \frac{x f(x)}{1-F(x)}=1$. Therefore, there exists a sequence of real numbers, $\left\{a_{N}\right\}_{N=1}^{\infty}$, such that $\frac{Y_{N}^{\star}(\mu)}{a_{N}}$ converges to a Frechet distributed random variable. The normalizing constants $a_{N}$ can be chosen to satisfy $F\left(a_{N}\right)=1-$ $\frac{1}{N}$. Hence, the asymptotic expansion of $a_{N}$ can be written as $a_{N}=\frac{N}{\mu\left(1-O\left(\frac{1}{N}\right)\right)}-\frac{1}{m \mu}$. The following lemma characterizes the asymptotic behavior of $F_{N}^{\star}(x)$.

Lemma 9: For $x=O(\log (N)), F_{N}^{\star}\left(a_{N} x\right)=$ $\mathrm{e}^{-m c_{N}(x)-O\left(\frac{c_{N}^{2}(x)}{N}\right)}$, where $c_{N}(x)$ is given by

$$
c_{N}(x)=\frac{1}{\frac{m x}{1-O\left(\frac{1}{N}\right)}-\frac{x}{N}+\frac{1}{N}} .
$$

Proof: Using the asymptotic expansion for $a_{N}, \frac{1}{m \mu a_{N} x+1}$ can be expanded as $\frac{1}{m \mu a_{N} x+1}=\frac{1}{N} \frac{\frac{m x}{\left(1-O\left(\frac{1}{N}\right)\right)}-\frac{x}{N}+\frac{1}{N}}{=}$ $\frac{1}{N} c_{N}(x)$. Hence, $F_{N}^{\star}\left(a_{N} x\right)$ can be expanded as $F_{N}^{\star}\left(a_{N} x\right)=$ $\left(1-\frac{1}{N} c_{N}(x)\right)^{m N}=\mathrm{e}^{-m c_{N}(x)-O\left(\frac{c_{N}^{2}(x)}{N}\right)}$.

Using Lemma 8 and following the same steps as in the throughput scaling proof given for $\operatorname{CoS}_{I L}^{1}$, we obtain

$$
\begin{aligned}
& \log \left(\frac{N}{\mu}\right)(1-\left.O\left(\frac{\log \log (N)}{\log (N)}\right)\right) \leq \tilde{R}_{\mathrm{IL}}^{4}(N, \mu) \\
& \leq \log \left(\frac{N}{\mu}\right)\left(1+O\left(\frac{\log \log (N)}{\log (N)}\right)\right)
\end{aligned}
$$


for any $\mu>0$. It also holds that $\liminf _{N \rightarrow \infty} \mu_{N}>0$. Since

$$
\tilde{R}_{\mathrm{IL}}^{4}\left(N, \frac{1}{Q_{\text {ave }}}\right) \leq R_{\mathrm{IL}}^{4}(N) \leq \tilde{R}_{\mathrm{IL}}^{4}(N, \epsilon)
$$

for $\epsilon>0$ small enough, we have $\lim _{N \rightarrow \infty} \frac{R_{\mathrm{IL}}^{4}(N)}{\log (N)}=1$.

Proof of Throughput Scaling in $\operatorname{CoS}_{\mathrm{IL}}^{5}$ : For any $\mu>0$, let $Y_{i}(\mu)=\frac{h_{i}}{\mu g_{i}}$ and $Y_{N}^{\star}(\mu)=\max _{1 \leq i \leq N} Y_{i}(\mu)$ as above. The CDF of $Y_{i}(\mu)$ is given by $F(x)=\frac{\mu x(K+1)}{\mu x(K+1)+1} \mathrm{e}^{-\frac{K}{\mu x(K+1)+1}}$ in this case. $F(x)$ satisfies $\lim _{x \rightarrow \infty} \frac{x f(x)}{1-F(x)}=1$. Therefore, there exists a sequence of real numbers, $\left\{a_{N}\right\}_{N=1}^{\infty}$, such that $\frac{Y_{N}^{\star}(\mu)}{a_{N}}$ converges to a Frechet distributed random variable. The normalizing constants $a_{N}$ can be chosen to satisfy $F\left(a_{N}\right)=$ $1-\frac{1}{N}$. Observe that $a_{N}$ tends to infinity and $\frac{1}{\mu a_{N}(K+1)+1}$ tends to zero as $N$ tends to infinity. We define $A_{N}=\frac{1}{\mu a_{N}(K+1)+1}$, and express $F\left(a_{N}\right)$ as (31) at the bottom of the page. Using (31), an asymptotic expansion for $a_{N}$ can be obtained as $a_{N}=$ $\frac{1}{\mu} N(1+o(1))-\frac{1}{\mu(K+1)}$. Now, we prove the next lemma that characterizes the asymptotic behavior of $F_{N}^{\star}(x)$, which is the $\mathrm{CDF}$ of $Y_{N}^{\star}(\mu)$.

Lemma 10: For $x=O(\log (N)), F^{\star}\left(a_{N} x\right)=$ $\mathrm{e}^{-(K+1) c_{N}(x)-O\left(\frac{c_{N}^{2}(x)}{N}\right)}$, where $c_{N}(x)$ is given by

$$
c_{N}(x)=\frac{1}{(K+1) x(1+o(1))-\frac{x}{N}+\frac{1}{N}} .
$$

Proof: Using the asymptotic expansion for $a_{N}$, $\frac{1}{\mu a_{N} x(K+1)+1}$ can be expanded as

$$
\begin{aligned}
\frac{1}{\mu a_{N}(K+1) x+1} & =\frac{1}{N} \frac{1}{(K+1) x(1+o(1))-\frac{x}{N}+\frac{1}{N}} \\
& =\frac{1}{N} c_{N}(x) .
\end{aligned}
$$

Hence, $F_{N}^{\star}\left(a_{N} x\right)$ can be expanded as $F_{N}^{\star}\left(a_{N} x\right)=$ $\left(1-\frac{1}{N} c_{N}(x)\right)^{N} \mathrm{e}^{-K c_{N}(x)}=\mathrm{e}^{-(K+1) c_{N}(x)-O\left(\frac{c_{N}^{2}(x)}{N}\right)}$.

Using Lemma 10 and following the same steps as in the throughput scaling proof given for $\operatorname{CoS}_{\mathrm{IL}}^{1}$, we obtain

$$
\begin{aligned}
& \log \left(\frac{1}{\mu} N\right)\left(1-O\left(\frac{\log \log (N)}{\log (N)}\right)\right)(1-o(1)) \\
& \leq \tilde{R}_{\mathrm{IL}}^{5}(N, \mu) \leq \log \left(\frac{1}{\mu} N\right)\left(1+O\left(\frac{\log \log (N)}{\log (N)}\right)\right)
\end{aligned}
$$

for any $\mu>0$. It also holds that $\liminf _{N \rightarrow \infty} \mu_{N}>0$. Since

$$
\tilde{R}_{\mathrm{IL}}^{5}\left(N, \frac{1}{Q_{\text {ave }}}\right) \leq R_{\mathrm{IL}}^{5}(N) \leq \tilde{R}_{\mathrm{IL}}^{5}(N, \epsilon)
$$

for $\epsilon>0$ small enough, we have $\lim _{N \rightarrow \infty} \frac{R_{\mathrm{IL}}^{5}(N)}{\log (N)}=1$.

\section{APPENDIX D \\ CONVERGENCE BEHAVIOR OF $\mu_{N}$}

In this appendix, we will establish an important convergence property for the Lagrange multipliers $\mu_{N}$ for all $\operatorname{CoS}_{\mathrm{IL}}^{i}, i \in$ $\{1,2, \ldots, 5\}$, in the next lemma. As we argue in Section IV, this property will imply that $Q_{\text {ave }}$ has logarithmic effects on the secondary network throughput.

Lemma 11: For all $\operatorname{CoS}_{\mathrm{IL}}^{i}, i \in\{1,2, \ldots, 5\}$, $\lim _{N \rightarrow \infty} \mu_{N}=\frac{1}{Q_{\text {ave }}}$.

Proof: Pick $\epsilon \stackrel{Q_{\text {ave }}}{>} 0$ such that $\liminf _{N \rightarrow \infty} \mu_{N} \geq \epsilon$. Then, $\left(1-\frac{1}{Y_{N}^{\star}\left(\frac{1}{Q_{\text {ave }}}\right)}\right)^{+} \leq\left(1-\frac{1}{X_{N}^{\star}}\right)^{+} \leq\left(1-\frac{1}{Y_{N}^{\star}(\epsilon)}\right)^{+}$for all $N$ large enough. By using the dominated convergence theorem, we have

$\lim _{N \rightarrow \infty} \mathrm{E}\left[\left(1-\frac{1}{Y_{N}^{\star}\left(\frac{1}{Q_{\text {ave }}}\right)}\right)^{+}\right]=\lim _{N \rightarrow \infty} \mathrm{E}\left[\left(1-\frac{1}{Y_{N}^{\star}(\epsilon)}\right)^{+}\right]=1$.

This implies that $\lim _{N \rightarrow \infty} \mathrm{E}\left[\left(1-\frac{1}{X_{N}^{\star}}\right)^{+}\right]=1$. The proof is complete since $\mu_{N}=\frac{1}{Q_{\text {ave }}} \mathrm{E}\left[\left(1-\frac{1}{X_{N}^{\star}}\right)^{+}\right]$.

\section{APPENDIX E}

\section{PROOF OF THEOREM 3}

Proof of Throughput Scaling in $\operatorname{CoS}_{\mathrm{PIL}}^{1-\mathrm{h}}$ : For PIL networks, we will start our analysis with the proof of the throughput scaling result in $\operatorname{CoS}_{\mathrm{PIL}}^{1-\mathrm{h}}$. For $\operatorname{CoS}_{\mathrm{PIL}}^{1-\mathrm{h}}$, the Rayleigh fading channel between the $i$ th SU and the SBS has a mean channel power gain $\alpha_{i}$, i.e., $\mathrm{E}\left[h_{i}\right]=\alpha_{i}$, whereas the Rician fading channel between the $i$ th SU and the PBS has a Rician factor $K_{i}$, and its mean channel power gain is equal to $\Omega_{i}$, i.e., $\mathrm{E}\left[g_{i}\right]=\Omega_{i}$. With a slight abuse of notation, the CDF of $X_{i, N}=\frac{h_{i}}{\lambda_{N}+\mu_{N} g_{i}}$ will be denoted by $F_{N}\left(x, \alpha_{i}, \Omega_{i}, K_{i}\right)$, and the CDF of $X_{N}^{\star}=\max _{1<i<N} X_{i, N}$ will be denoted by $F_{N}^{\star}(x) .^{2}$ We let $\alpha_{\max }=\sup _{i>1} \alpha_{i}, \alpha_{\min }=\inf _{i>1} \alpha_{i}$, $\Omega_{\max }=\sup _{i>1} \Omega_{i}, \Omega_{\min }=\inf _{i \geq 1} \Omega_{i}, K_{\max }=\sup _{i>1} K_{i}$ and $K_{\min }=\inf _{i \geq 1} K_{i}$. It is assumed that $\alpha_{\min }>0, \Omega_{\min }>0$, $K_{\min } \geq 0, \alpha_{\max }<\infty, \Omega_{\max }<\infty$ and $K_{\max }<\infty$.

After some analysis, $F_{N}\left(x, \alpha_{i}, \Omega_{i}, K_{i}\right)$ can be derived as

$$
\begin{aligned}
& F_{N}\left(x, \alpha_{i}, \Omega_{i}, K_{i}\right) \\
& \quad=1-\frac{\alpha_{i}\left(K_{i}+1\right)}{\mu_{N} \Omega_{i} x+\alpha_{i}\left(K_{i}+1\right)} \mathrm{e}^{-x\left(\frac{\lambda_{N}}{\alpha_{i}}+\frac{\mu_{N} \Omega_{i} K_{i}}{\mu_{N} \Omega_{i} x+\alpha_{i}\left(K_{i}+1\right)}\right)} .
\end{aligned}
$$

${ }^{2}$ The notation used to represent the CDFs of $X_{i, N}$ and $X_{N}^{\star}$ should not be confused with the notation used to represent the CDFs of the auxiliary random variables $Y_{i}(\lambda, \mu)$ and $Y_{N}^{\star}(\lambda, \mu)$ introduced in previous appendixes.

$$
\begin{aligned}
F\left(a_{N}\right) & =1-(K+1) A_{N}\left(1-\frac{K A_{N}}{K+1} \sum_{k=1}^{\infty} \frac{\left(-K A_{N}\right)^{k-1}}{k !}-\frac{K^{2} A_{N}}{K+1} \sum_{k=2}^{\infty} \frac{\left(-K A_{N}\right)^{k-2}}{k !}\right) \\
& =1-(K+1) A_{N}(1+o(1)) .
\end{aligned}
$$


Then, $F_{N}^{\star}(x)$ is equal to $F_{N}^{\star}(x)=\prod_{i=1}^{N} F_{N}\left(x, \alpha_{i}, \Omega_{i}, K_{i}\right)$. After some calculations, we can show that $\frac{\partial F_{N}\left(x, \alpha_{i}, \Omega_{i}, K_{i}\right)}{\partial \alpha_{i}} \leq 0$, $\frac{\partial F_{N}\left(x, \alpha_{i}, \Omega_{i}, K_{i}\right)}{\partial \Omega_{i}} \geq 0$ and $\frac{\partial F_{N}\left(x, \alpha_{i}, \Omega_{i}, K_{i}\right)}{\partial K_{i}} \geq 0$, which implies that $F_{N}\left(x, \alpha_{i}, \Omega_{i}, K_{i}\right)$ is a decreasing function of $\alpha_{i}$, and an increasing function of $\Omega_{i}$ and $K_{i}$. Hence, we have

$$
\begin{aligned}
\left(F _ { N } \left(x, \alpha_{\max }, \Omega_{\min },\right.\right. & \left.\left.K_{\min }\right)\right)^{N} \leq F_{N}^{\star}(x) \\
& \leq\left(F_{N}\left(x, \alpha_{\min }, \Omega_{\max }, K_{\max }\right)\right)^{N} .
\end{aligned}
$$

Under some mild conditions on the tails of $F_{N}^{\star}(x)^{3}$, the sum-rate can be written as

$$
R_{\mathrm{PIL}}^{1-\mathrm{h}}(N)=\int_{1}^{\infty} \frac{1-F_{N}^{\star}(x)}{x} d x
$$

Using (36) and (37), $R_{\mathrm{PIL}}^{1-\mathrm{h}}(N)$ can be upper and lower bounded as

$$
\begin{aligned}
R_{N}^{\mathrm{L}} & =\int_{1}^{\infty} \frac{1-\left(F_{N}\left(x, \alpha_{\min }, \Omega_{\max }, K_{\max }\right)\right)^{N}}{x} d x \\
& \leq R_{\mathrm{PIL}}^{1-\mathrm{h}}(N) \leq \\
R_{N}^{\mathrm{U}} & =\int_{1}^{\infty} \frac{1-\left(F_{N}\left(x, \alpha_{\max }, \Omega_{\min }, K_{\min }\right)\right)^{N}}{x} d x .
\end{aligned}
$$

Observe that $R_{N}^{\mathrm{U}}$ is the sum-rate of a cognitive radio secondary network in which STSB channels are Rayleigh fading channels with the same mean channel power gain $\alpha_{\max }$, and STPB channels are Rician fading channels with the same Rician factor $K_{\min }$ and the same mean channel power gain $\Omega_{\min }$. Similarly, $R_{N}^{\mathrm{L}}$ is the sum-rate of a cognitive radio secondary network in which STSB channels are Rayleigh fading channels with the same mean channel power gain $\alpha_{\min }$, and STPB channels are Rician fading channels with the same Rician factor $K_{\max }$ and the same mean channel power gain $\Omega_{\max }$. Following the similar steps that we used to prove throughput scaling in $\operatorname{CoS}_{\mathrm{PIL}}^{1}$, it is easy to prove that $R_{N}^{\mathrm{L}}$ and $R_{N}^{\mathrm{U}}$ have $\log \log (N)$-type behavior. Therefore, we have

$$
\begin{aligned}
\lim _{N \rightarrow \infty} \frac{R_{N}^{\mathrm{L}}}{\log \log (N)} & =\lim _{N \rightarrow \infty} \frac{R_{N}^{\mathrm{U}}}{\log \log (N)} \\
& =\lim _{N \rightarrow \infty} \frac{R_{\mathrm{PIL}}^{1-\mathrm{h}}(N)}{\log \log (N)}=1 .
\end{aligned}
$$

Proof of Throughput Scaling in $\mathrm{CoS}_{\mathrm{PIL}}^{3-\mathrm{h}}$ : In this case, the Rayleigh fading channel between the $i$ th SU and the SBS has a mean channel power gain $\alpha_{i}$, i.e., $\mathrm{E}\left[h_{i}\right]=\alpha_{i}$, whereas the Nakagami fading channel between the $i$ th $\mathrm{SU}$ and the PBS has a Nakagami parameter $m_{i}\left(m_{i} \geq 0.5\right)$, and its mean channel power gain is equal to $\Omega_{i}$, i.e., $\mathrm{E}\left[g_{i}\right]=\Omega_{i}$. The similar assumptions on $\left\{\alpha_{i}\right\}_{i=1}^{N},\left\{\Omega_{i}\right\}_{i=1}^{N}$ and $\left\{m_{i}\right\}_{i=1}^{N}$ above also hold in this case without loss of any generality. The $\mathrm{CDF}$ of $X_{i, N}=\frac{h_{i}}{\lambda_{N}+\mu_{N} g_{i}}$ is given by $F_{N}\left(x, \alpha_{i}, \Omega_{i}, m_{i}\right)=$ $1-\mathrm{e}^{-\frac{\lambda_{N}}{\alpha_{i}} x}\left(\frac{\alpha_{i} m_{i}}{\mu_{N} \Omega_{i} x+\alpha_{i} m_{i}}\right)^{m_{i}}$. After some calculations, we

\footnotetext{
${ }^{3}$ The required condition is $\lim _{x \rightarrow \infty}\left(1-F_{N}^{\star}(x)\right) \log (x)=0$ for all finite $N$, which is satisfied by the distributions of interest in this paper.
}

can show that $\frac{\partial F_{N}\left(x, \alpha_{i}, \Omega_{i}, m_{i}\right)}{\partial \alpha_{i}} \leq 0, \frac{\partial F_{N}\left(x, \alpha_{i}, \Omega_{i}, m_{i}\right)}{\partial \Omega_{i}} \geq 0$ and $\frac{\partial F_{N}\left(x, \alpha_{i}, \Omega_{i}, m_{i}\right)}{\partial m_{i}} \geq 0$. Hence, we have

$$
\begin{aligned}
\left(F _ { N } \left(x, \alpha_{\max }, \Omega_{\min },\right.\right. & \left.\left.m_{\min }\right)\right)^{N} \leq F_{N}^{\star}(x) \\
& \leq\left(F_{N}\left(x, \alpha_{\min }, \Omega_{\max }, m_{\max }\right)\right)^{N}
\end{aligned}
$$

where $F_{N}^{\star}(x)$ is the CDF of $X_{N}^{\star}=\max _{1 \leq i \leq N} X_{i, N}$, and $\alpha_{\max }$, $\alpha_{\min }, \Omega_{\max }, \Omega_{\min }, m_{\max }$ and $m_{\min }$ are defined as above. Using (39), $R_{\mathrm{PII}}^{3-\mathrm{h}}(N)$ can be upper and lower bounded as

$$
\begin{aligned}
R_{N}^{\mathrm{L}} & =\int_{1}^{\infty} \frac{1-\left(F_{N}\left(x, \alpha_{\min }, \Omega_{\max }, m_{\max }\right)\right)^{N}}{x} d x \\
& \leq R_{\mathrm{PIL}}^{3-\mathrm{h}}(N) \\
\leq R_{N}^{\mathrm{U}} & =\int_{1}^{\infty} \frac{1-\left(F_{N}\left(x, \alpha_{\max }, \Omega_{\min }, m_{\min }\right)\right)^{N}}{x} d x .
\end{aligned}
$$

Following our earlier analysis for homogeneous CoSs, it is easy to show that $R_{N}^{\mathrm{L}}$ and $R_{N}^{\mathrm{U}}$ scale according to $\log \log (N)$, which implies $\lim _{N \rightarrow \infty} \frac{R_{\mathrm{PL}}^{3-\mathrm{h}}(N)}{\log \log (N)}=1$.

\section{APPENDIX F \\ PROOF OF THEOREM 4}

Proof of Throughput Scaling in $\mathrm{CoS}_{\mathrm{IL}}^{1-\mathrm{h}}$ : For IL networks, we will start our analysis with the proof of the throughput scaling result in $\operatorname{CoS}_{\mathrm{IL}}^{1-\mathrm{h}}$. For $\operatorname{CoS}_{\mathrm{IL}}^{1-\mathrm{h}}$, the Rayleigh fading channel between the $i$ th $\mathrm{SU}$ and the SBS has a mean channel power gain $\alpha_{i}$, i.e., $\mathrm{E}\left[h_{i}\right]=\alpha_{i}$, whereas the Rician fading channel between the $i$ th SU and the PBS has a Rician factor $K_{i}$, and its mean channel power gain is equal to $\Omega_{i}$, i.e., $\mathrm{E}\left[g_{i}\right]=\Omega_{i}$. It is easy to show that the CDF of $X_{i, N}=\frac{h_{i}}{\mu_{N} g_{i}}$ is given by $F_{N}\left(x, \alpha_{i}, \Omega_{i}, K_{i}\right)=$ $1-\frac{\alpha_{i}\left(K_{i}+1\right)}{\mu_{N} \Omega_{i} x+\alpha_{i}\left(K_{i}+1\right)} \mathrm{e}^{-\left(\frac{\mu_{N} \Omega_{i} K_{i} x}{\mu_{N} \Omega_{i} x+\alpha_{i}\left(K_{i}+1\right)}\right)}$, and the following inequalities

$$
\begin{aligned}
& \left(F_{N}\left(x, \alpha_{\max }, \Omega_{\min }, K_{\min }\right)\right)^{N} \\
& \quad \leq F_{N}^{\star}(x) \leq\left(F_{N}\left(x, \alpha_{\min }, \Omega_{\max }, K_{\max }\right)\right)^{N}
\end{aligned}
$$

hold, where $F_{N}^{\star}(x)$ is the CDF of $X_{N}^{\star}=\max _{1 \leq i \leq N} X_{i, N}$, and $\alpha_{\max }, \alpha_{\min }, \Omega_{\max }, \Omega_{\min }, K_{\max }$ and $K_{\min }$ are defined as above. The same assumptions above on these system parameters continue to hold for IL networks too, but $K_{\max }<\infty$ is now a necessary assumption to make use of multiuser diversity between SUs and the PBS. Using (41), $R_{\mathrm{IL}}^{1-\mathrm{h}}(N)$ can be upper and lower bounded as

$$
\begin{aligned}
R_{N}^{\mathrm{L}} & =\int_{1}^{\infty} \frac{1-\left(F_{N}\left(x, \alpha_{\min }, \Omega_{\max }, K_{\max }\right)\right)^{N}}{x} d x \\
& \leq R_{\mathrm{IL}}^{1-\mathrm{h}}(N) \leq \\
R_{N}^{\mathrm{U}} & =\int_{1}^{\infty} \frac{1-\left(F_{N}\left(x, \alpha_{\max }, \Omega_{\min }, K_{\min }\right)\right)^{N}}{x} d x .
\end{aligned}
$$

Following our earlier analysis for homogeneous CoSs, it is easy to show that $R_{N}^{\mathrm{L}}$ and $R_{N}^{\mathrm{U}}$ scale according to $\log (N)$, which implies $\lim _{N \rightarrow \infty} \frac{R_{\mathrm{I}}^{1-\mathrm{h}}(N)}{\log (N)}=1$.

Proof of Throughput Scaling in $\mathrm{CoS}_{\mathrm{IL}}^{3-\mathrm{h}}$ : In this case, the Rayleigh fading channel between the $i$ th SU and the SBS has a mean channel power gain $\alpha_{i}$, i.e., $\mathrm{E}\left[h_{i}\right]=\alpha_{i}$, whereas the 
Nakagami fading channel between the $i$ th SU and the PBS has a Nakagami parameter $m_{i}\left(m_{i} \geq 0.5\right)$, and its mean channel power gain is equal to $\Omega_{i}$, i.e., $\mathrm{E}\left[g_{i}\right]=\Omega_{i}$. It is easy to show that the $\mathrm{CDF}$ of $X_{i, N}=\frac{h_{i}}{\mu_{N} g_{i}}$ is given by $F_{N}\left(x, \alpha_{i}, \Omega_{i}, m_{i}\right)=$ $1-\left(\frac{\alpha_{i} m_{i}}{\mu_{N} \Omega_{i} x+\alpha_{i} m_{i}}\right)^{m_{i}}$, and the following inequalities

$$
\begin{aligned}
& \left(F_{N}\left(x, \alpha_{\max }, \Omega_{\min }, m_{\min }\right)\right)^{N} \\
& \quad \leq F_{N}^{\star}(x) \leq\left(F_{N}\left(x, \alpha_{\min }, \Omega_{\max }, m_{\max }\right)\right)^{N}
\end{aligned}
$$

hold, where $F_{N}^{\star}(x)$ is the CDF of $X_{N}^{\star}=\max _{1 \leq i \leq N} X_{i, N}$, and $\alpha_{\max }, \alpha_{\min }, \Omega_{\max }, \Omega_{\min }, m_{\max }$ and $m_{\min }$ are defined as above. The same assumptions above on these system parameters continue to hold in this case, too. Using (43), we have

$$
\begin{aligned}
R_{N}^{\mathrm{L}} & =\int_{1}^{\infty} \frac{1-\left(F_{N}\left(x, \alpha_{\min }, \Omega_{\max }, m_{\max }\right)\right)^{N}}{x} d x \\
& \leq R_{\mathrm{IL}}^{3-\mathrm{h}}(N) \leq \\
R_{N}^{\mathrm{U}} & =\int_{1}^{\infty} \frac{1-\left(F_{N}\left(x, \alpha_{\max }, \Omega_{\min }, m_{\min }\right)\right)^{N}}{x} d x .
\end{aligned}
$$

Following our earlier analysis for homogeneous CoSs, it is easy to show that $R_{N}^{\mathrm{L}}$ and $R_{N}^{\mathrm{U}}$ scale according to $\frac{1}{m_{\max }} \log (N)$ and $\frac{1}{m_{\min }} \log (N)$, respectively. Hence, we have $\frac{1}{m_{\max }} \leq$ $\liminf _{N \rightarrow \infty} \frac{R_{\mathrm{IL}}^{3-\mathrm{h}}(N)}{\log (N)} \leq \lim \sup _{N \rightarrow \infty} \frac{R_{\mathrm{I}}^{3-\mathrm{h}}(N)}{\log (N)} \leq \frac{1}{m_{\min }}$.

Proof of Throughput Scaling in $\mathrm{CoS}_{\mathrm{IL}}^{4-\mathrm{h}}$ : In this case, the Nakagami fading channel between the $i$ th SU and the SBS has a Nakagami parameter $m_{i}\left(m_{i} \geq 0.5\right)$ and a mean channel power gain $\Omega_{i}$, i.e., $\mathrm{E}\left[h_{i}\right]=\Omega_{i}$, whereas the Rayleigh fading channel between the $i$ th SU and the PBS has a mean channel power gain $\alpha_{i}$, i.e., $\mathrm{E}\left[g_{i}\right]=\alpha_{i}$. It is easy to show that the $\mathrm{CDF}$ of $X_{i, N}=$ $\frac{h_{i}}{\mu_{N} g_{i}}$ is given by $F_{N}\left(x, \alpha_{i}, \Omega_{i}, m_{i}\right)=\left(\frac{\mu_{N} \alpha_{i} m_{i} x}{\mu_{N} \alpha_{i} m_{i} x+\Omega_{i}}\right)^{m_{i}}$, and the following inequalities:

$$
\begin{aligned}
& \left(F_{N}\left(x, \alpha_{\min }, \Omega_{\max }, m_{\max }\right)\right)^{N} \\
& \quad \leq F_{N}^{\star}(x) \leq\left(F_{N}\left(x, \alpha_{\max }, \Omega_{\min }, m_{\min }\right)\right)^{N}
\end{aligned}
$$

hold, where $F_{N}^{\star}(x)$ is the CDF of $X_{N}^{\star}=\max _{1 \leq i \leq N} X_{i, N}$, and $\alpha_{\max }, \alpha_{\min }, \Omega_{\max }, \Omega_{\min }, m_{\max }$ and $m_{\min }$ are defined as above. The same assumptions above on these system parameters continue to hold in this case, too. Using (45), $R_{\mathrm{IL}}^{4-\mathrm{h}}(N)$ can be upper and lower bounded as

$$
\begin{aligned}
R_{N}^{\mathrm{L}} & =\int_{1}^{\infty} \frac{1-\left(F_{N}\left(x, \alpha_{\max }, \Omega_{\min }, m_{\min }\right)\right)^{N}}{x} d x \\
& \leq R_{\mathrm{IL}}^{4-\mathrm{h}}(N) \leq \\
R_{N}^{\mathrm{U}} & =\int_{1}^{\infty} \frac{1-\left(F_{N}\left(x, \alpha_{\min }, \Omega_{\max }, m_{\max }\right)\right)^{N}}{x} d x .
\end{aligned}
$$

Following our earlier analysis for homogeneous CoSs, it is easy to show that $R_{N}^{\mathrm{L}}$ and $R_{N}^{\mathrm{U}}$ scale according to $\log (N)$, which implies $\lim _{N \rightarrow \infty} \frac{R_{\mathrm{IL}}^{4-\mathrm{h}}(N)}{\log (N)}=1$.

Proof of Throughput Scaling in $\mathrm{CoS}_{\mathrm{IL}}^{5-\mathrm{h}}$ : In this case, the Rician fading channel between the $i$ th $\mathrm{SU}$ and the SBS has a Rician factor $K_{i}$ and a mean channel power gain $\Omega_{i}$, i.e., $\mathrm{E}\left[h_{i}\right]=$ $\Omega_{i}$, whereas the Rayleigh fading channel between the $i$ th SU and the PBS has a mean channel power gain $\alpha_{i}$, i.e., $\mathrm{E}\left[g_{i}\right]=\alpha_{i}$. It is easy to show that the CDF of $X_{i, N}=\frac{h_{i}}{\mu_{N} g_{i}}$ is given by $F_{N}\left(x, \alpha_{i}, \Omega_{i}, K_{i}\right)=\frac{\mu_{N} \alpha_{i}\left(K_{i}+1\right) x}{\mu_{N} \alpha_{i}\left(K_{i}+1\right) x+\Omega_{i}} \mathrm{e}^{-\left(\frac{K_{i} \Omega_{i}}{\mu_{N} \alpha_{i}\left(K_{i}+1\right) x+\Omega_{i}}\right)}$, and the following inequalities:

$$
\begin{aligned}
& \left(F_{N}\left(x, \alpha_{\min }, \Omega_{\max }, K_{\max }\right)\right)^{N} \\
& \quad \leq F_{N}^{\star}(x) \leq\left(F_{N}\left(x, \alpha_{\max }, \Omega_{\min }, K_{\min }\right)\right)^{N}
\end{aligned}
$$

hold, where $F_{N}^{\star}(x)$ is the CDF of $X_{N}^{\star}=\max _{1 \leq i \leq N} X_{i, N}$, and $\alpha_{\max }, \alpha_{\min }, \Omega_{\max }, \Omega_{\min }, K_{\max }$ and $K_{\min }$ are defined as above. The same assumptions above on these system parameters continue to hold in this case, too. Using (47), $R_{\mathrm{IL}}^{5-\mathrm{h}}(N)$ can be upper and lower bounded as

$$
\begin{aligned}
R_{N}^{\mathrm{L}} & =\int_{1}^{\infty} \frac{1-\left(F_{N}\left(x, \alpha_{\max }, \Omega_{\min }, K_{\min }\right)\right)^{N}}{x} d x \\
& \leq R_{\mathrm{IL}}^{5-\mathrm{h}}(N) \\
\leq R_{N}^{\mathrm{U}} & =\int_{1}^{\infty} \frac{1-\left(F_{N}\left(x, \alpha_{\min }, \Omega_{\max }, K_{\max }\right)\right)^{N}}{x} d x .
\end{aligned}
$$

Following our earlier analysis for homogeneous CoSs, it is easy to show that $R_{N}^{\mathrm{L}}$ and $R_{N}^{\mathrm{U}}$ scale according to $\log (N)$, which implies $\lim _{N \rightarrow \infty} \frac{R_{\mathrm{IL}}^{5-\mathrm{h}}(N)}{\log (N)}=1$.

\section{REFERENCES}

[1] I. F. Akyildiz, W.-Y. Lee, M. C. Vuran, and S. Mohanty, "NeXt generation/dynamic spectrum access/cognitive radio wireless networks: A survey," Comput. Netw., vol. 50, no. 13, pp. 2127-2159, Sep. 2006.

[2] J. Mitola, III, "Cognitive radio for flexible mobile multimedia communications," in Proc. IEEE Int. Workshop Mobile Multimedia Commun., San Diego, CA, Nov. 1999, pp. 3-10.

[3] J. Mitola, III and G. Q. Maguire, Jr., "Cognitive radio: Making software radios more personal," IEEE Pers. Commun., vol. 6, no. 4, pp. 13-18, Aug. 1999.

[4] A. Goldsmith, S. A. Jafar, I. Maric, and S. Srinivasa, "Breaking spectrum gridlock with cognitive radios: An information theoretic perspective," Proc. IEEE, vol. 97, no. 5, pp. 894-914, May 2009.

[5] FCC Spectrum Policy Task Force, "Report of the Spectrum Efficiency Working Group," Tech. Rep. 02-135, Nov. 2002 [Online]. Available: http://www.fcc.gov/sptf/files/SEWGFinalReport_1.pdf

[6] P. J. Kolodzy, "Interference temperature: A metric for dynamic spectrum utilization," Int. J. Netw. Manage., vol. 16, no. 2, pp. 103-113, Mar. 2006.

[7] M. Gastpar, "On capacity under receive and spatial spectrum-sharing constraints," IEEE Trans. Inf. Theory, vol. 53, no. 2, pp. 471-487, Feb. 2007.

[8] A. Ghasemi and E. S. Sousa, "Fundamental limits of spectrum-sharing in fading environments," IEEE Trans. Wireless Commun., vol. 6, no. 2, pp. 649-658, Feb. 2007

[9] X. Kang, Y. Liang, A. Nallanathan, H. K. Garg, and R. Zhang, "Optimal power allocation for fading channels in cognitive radio networks: Ergodic capacity and outage capacity," IEEE Trans. Wireless Commun., vol. 8, no. 2, pp. 940-950, Feb. 2009.

[10] R. Zhang, S. Cui, and Y.-C. Liang, "On ergodic sum capacity of fading cognitive multiple-access and broadcast channels," IEEE Trans. Inf. Theory, vol. 55, no. 11, pp. 5161-5178, Nov. 2009.

[11] R. Zhang, Y.-C. Liang, and S. Cui, "Dynamic resource allocation in cognitive radio networks," IEEE Signal Process. Mag., vol. 27, no. 3 , pp. 102-114, Mar. 2010

[12] R. Knopp and P. A. Humblet, "Information capacity and power control in single cell multiuser communications," in Proc. IEEE Int. Conf. Commun., Seattle, WA, Jun. 1995, vol. 1, pp. 331-335.

[13] D. N. C. Tse and S. V. Hanly, "Multiaccess fading channels-part I Polymatroid structure, optimal resource allocation and throughput capacities," IEEE Trans. Inf. Theory, vol. 44, no. 7, pp. 2796-2815, Nov. 1998.

[14] P. Viswanath, D. N. C. Tse, and R. Laroia, "Opportunistic beamforming using dumb antennas," IEEE Trans. Inf. Theory, vol. 48, no. 6, pp. 1277-1294, Jun. 2002

[15] E. Biglieri, J. Proakis, and S. Shamai, "Fading channels: Informationtheoretic and communication aspects," IEEE Trans. Inf. Theory, vol. 44, no. 6, pp. 2619-2692, Oct. 1998 
[16] H. A. Suraweera, J. Gao, P. J. Smith, M. Shafi, and M. Faulkner, "Channel capacity limits of cognitive radio in asymmetric fading environment," in Proc. IEEE Int. Conf. Commun. (ICC), Beijing, China, May 2008, pp. 4048-4053.

[17] W. Yu, "Uplink-downlink duality via minimax duality," IEEE Trans. Inf. Theory, vol. 52, pp. 361-374, Feb. 2006.

[18] G. A. Gupta and S. Toumpis, "Power allocation over parallel Gaussian multiple access and broadcast channels," IEEE Trans. Inf. Theory, vol. 52, pp. 3274-3282, Jul. 2006.

[19] M. Shariff and B. Hassibi, "On the capacity of MIMO broadcast channels with partial side information," IEEE Trans. Inf. Theory, vol. 51, p. 506522, Feb. 2005.

[20] J. Diaz, O. Simeone, and Y. B. Ness, "Asymptotic analysis of reducedfeedback strategies for MIMO Gaussian broadcast channels," IEEE Trans. Inf. Theory, vol. 54, no. 3, pp. 1308-1316, Mar. 2008.

[21] M. Pugh and B. D. Rao, "Reduced feedback schemes using random beamforming in MIMO broadcast channels," IEEE Trans. Signal Process., vol. 58, no. 3, pp. 1821-1832, Mar. 2010.

[22] H. A. David and H. N. Nagaraja, Order Statistics. New York: Wiley, 2003.

[23] H. Wang, J. Lee, S. Kim, and D. Hong, "Capacity of secondary users exploiting multispectrum and multiuser diversity in spectrum-sharing environments," IEEE Trans. Veh. Technol., vol. 59, no. 2, pp. 1030-1036, Feb. 2010.

[24] A. Tajer and X. Wang, "Multiuser diversity gain in cognitive networks," IEEE/ACM Trans. Netw., vol. 18, no. 6, pp. 1766-1779, Dec. 2010.

[25] T. W. Ban, W. Choi, B. C. Jung, and D. K. Sung, "Multi-user diversity in a spectrum sharing system," IEEE Trans. Wireless Commun., vol. 8, no. 1, pp. 102-106, Jan. 2009.

[26] R. Zhang and Y.-C. Liang, "Investigation on multiuser diversity in spectrum sharing based cognitive radio networks," IEEE Commun. Lett., vol. 14, no. 2, pp. 133-135, Feb. 2010.

[27] N. Devroye, M. Vu, and V. Tarokh, "Achievable rates and scaling laws for cognitive radio channels," EURASIP J. Wireless Commun. Netw., Special Issue on Cognitive Radio and Dynamic Spectrum Sharing Systems, pp. 1-12, Jan. 2008.

[28] C. Yin, L. Gao, and S. Cui, "Scaling laws of overlaid wireless networks: A cognitive radio network versus A primary network," IEEE/ACM Trans. Netw., vol. 18, no. 4, pp. 1317-1329, Aug. 2010.

[29] P. Gupta and P. R. Kumar, "The capacity of wireless networks," IEEE Trans. Inf. Theory, vol. 46, no. 2, pp. 388-404, Mar. 2000.

[30] V. R. Cadambe and S. A. Jafar, "Interference alignment and degrees of freedom of the K-user interference channel," IEEE Trans. Inf. Theory, vol. 54, no. 8, pp. 3425-3441, Aug. 2008.

[31] G. Stüber, Principles of Mobile Communication. Boston, MA: Kluwer Academic, 1996.

[32] D. G. Luenberger, Optimization by Vector Space Methods. New York: Wiley, 1968.

[33] P. Billingsley, Probability and Measure, third ed. New York: Wiley, 1995.

[34] D. Tse and P. Viswanath, Fundamentals of Wireless Communication. Cambridge, U.K.: Cambridge Univ. Press, 2005.

[35] I. S. Gradshteyn and I. M. Ryzhik, Table of Integrals, Series and Products, 6th ed. New York: Academic, 2000.

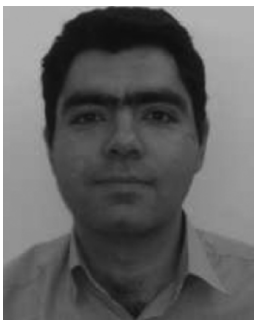

Ehsan Nekouei (S'11) received the B.S. degree from Shahid Bahonar University of Kerman, Kerman, Iran, in 2003 and the M.S. degree from Tarbiat Modares University, Tehran, Iran, in 2006. He is working towards the Ph.D. degree in the Department of Electrical and Electronic Engineering, University of Melbourne.

His research interests include wireless communications and information theory.

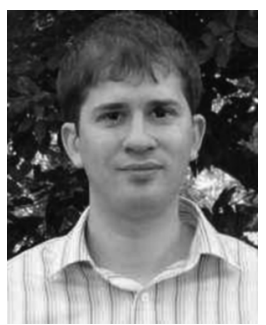

Hazer Inaltekin (S'04-M'06) received the B.S. degree (with High Hons.) in electrical and electronics engineering from Bogazici University, Istanbul, Turkey, in 2001 and the M.S./Ph.D. degree in electrical and computer engineering from Cornell University, Ithaca, NY, in 2006.

He was a Postdoctoral Research Associate at Cornell University, from 2006 to 2007 and at Princeton University, Princeton, NJ, from 2007 to 2009. In 2009, he joined the Department of Electrical and Electronic Engineering at the University of Melbourne as a Research Fellow. He is currently an Assistant Professor in the Department of Electrical and Electronics Engineering at Antalya International University, Antalya, Turkey. His research interests include wireless communications, wireless networks, social networks, game theory, and information theory.

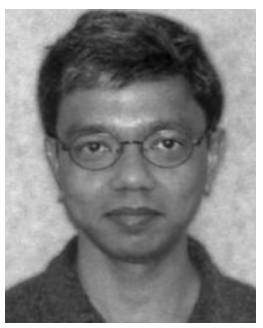

Subhrakanti Dey (SM'06) was born in India in 1968. He received the B.Tech. and M.Tech. degrees from the Department of Electronics and Electrical Communication Engineering, Indian Institute of Technology, Kharagpur, India, in 1991 and 1993, respectively, and the Ph.D. degree from the Department of Systems Engineering, Research School of Information Sciences and Engineering, Australian National University, Canberra, Australia, in 1996.

Since February 2000, he has been with the Department of Electrical and Electronic Engineering, University of Melbourne, Parkville, Australia, where he is currently a full Professor. From September 1995 to September 1997 and September 1998 to February 2000, he was a Postdoctoral Research Fellow with the Department of Systems Engineering, Australian National University. From September 1997 to September 1998, he was a Postdoctoral Research Associate with the Institute for Systems Research, University of Maryland, College Park. His current research interests include networked control systems, wireless communications and networks, signal processing for sensor networks, and stochastic and adaptive estimation and control.

Prof. Dey currently serves on the Editorial Board of the Elsevier Systems and Control Letters. He was also an Associate Editor for the IEEE TRANSACTIONS on Signal Processing and the IEEE TRAnsactions on Automatic CONTROL. 